\title{
Some operations on hesitant fuzzy soft sets over UP-algebras
}

Phakawat Mosrijai, Aiyared lampan*

Department of Mathematics, School of Science, University of Phayao, Phayao 56000, Thailand.

\begin{abstract}
This paper aim is to apply the notions of the (restricted) union, the (extended) intersection, the AND, and the OR of any hesitant fuzzy soft set to UP-algebras. Moreover, we discuss results of the intersection and the union of hesitant fuzzy sets on UP-algebras and also apply the notions of the hesitant union and the hesitant intersection of hesitant fuzzy sets to UP-algebras.
\end{abstract}

Keywords: UP-algebra, hesitant fuzzy soft set, anti-hesitant fuzzy soft set, hesitant union, hesitant intersection.

2010 MSC: 03G25.

(C)2020 All rights reserved.

\section{Introduction}

The branch of the logical algebra, UP-algebras were introduced by Iampan [5] in 2017, and it is known that the class of KU-algebras [21] is a proper subclass of the class of UP-algebras. It have been examined by several researchers, for example, Somjanta et al. [28] introduced the notion of fuzzy sets in UP-algebras, the notion of intuitionistic fuzzy sets in UP-algebras was introduced by Kesorn et al. [9], Kaijae et al. [8] introduced the notions of anti-fuzzy UP-ideals and anti-fuzzy UP-subalgebras of UP-algebras, the notion of Q-fuzzy sets in UP-algebras was introduced by Tanamoon et al. [31], Sripaeng et al. [30] introduced the notion anti Q-fuzzy UP-ideals and anti Q-fuzzy UP-subalgebras of UP-algebras, the notion of $\mathcal{N}$-fuzzy sets in UP-algebras was introduced by Songsaeng and Iampan [29], Senapati et al. [26, 27] applied cubic set and interval-valued intuitionistic fuzzy structure in UP-algebras, Romano [22] introduced the notion of proper UP-filters in UP-algebras, etc..

A soft set over a universe set is a parametized family of subsets of the universe set. Molodtsov [14] introduced the concept of soft sets over a universe set in 1999. The soft set theory was a new mathematical tool to handle uncertainty but the classical soft sets are not appropriate to deal with some imprecisions. The soft set model has been combined with other mathematical models. For instance, fuzzy soft sets [10] are based on a combination of fuzzy sets and soft sets, intuitionistic fuzzy soft sets [11,13] are combined by intuitionistic fuzzy sets and soft sets, interval-valued fuzzy soft sets [34], and so on. By the way, Maji

\footnotetext{
*Corresponding author

Email addresses: phakawat.mo@gmail.com (Phakawat Mosrijai), aiyared.ia@up.ac.th (Aiyared Iampan)

doi: $10.22436 /$ jmcs.020.02.06
}

Received: 2019-03-03 Revised: 2019-09-07 Accepted: 2019-09-16 
et al. [12] defined equality of two soft sets, subset and superset of a soft set, complement of a soft set, null soft set, and absolute soft set in 2003. Moreover, they also introduced the concepts of the AND operation and the OR operation on two soft sets, and the union and the intersection of two soft sets. Ali et al. [1] latterly introduced the others such as the restricted intersection, the restricted union, the restricted difference, and the extended intersection of two soft sets.

A hesitant fuzzy set on a set is a function from a reference set to a power set of the unit interval. The concept of a hesitant fuzzy set on a set was first considered by Torra [32] in 2010. The hesitant fuzzy set, which can be perfectly described in terms of the opinions of decision makers is a very useful tool to deal with uncertainty. The hesitant fuzzy set theories developed by Torra and others have found many applications in the domain of mathematics, for example, Jun [7] defined the hesitant union, and the hesitant intersection of two hesitant fuzzy sets, etc.. In UP-algebras, Mosrijai et al. [19] extended the concept of fuzzy sets in UP-algebras to hesitant fuzzy sets on UP-algebras, and Satirad et al. [25] considered level subsets of a hesitant fuzzy set on UP-algebras in 2017. The concept of partial constant hesitant fuzzy sets on UP-algebras was introduced by Mosrijai et al. [20] afterwards. Mosrijai and Iampan [15] introduced the notion of anti-type of hesitant fuzzy sets on UP-algebras in 2018.

The concept of hesitant fuzzy soft sets that is a link between classical soft sets and hesitant fuzzy sets was introduced by Babitha and John [3] in 2013. Wang et al. [33] applied the hesitant fuzzy soft set theory to a decision making problem in 2014 and they also defined the complement, the "AND", the "OR", the union, and the intersection operations on hesitant fuzzy soft set. In UP-algebras, Mosrijai and Iampan [16] extended the concept of hesitant fuzzy sets on UP-algebras to hesitant fuzzy soft sets over UP-algebras, and they also introduced the notion of hesitant fuzzy soft sets over UP-algebras by mean of anti-type (see [17]).

In this paper, we study about some operations that can be applied to theories of hesitant fuzzy sets on UP-algebras or theories of hesitant fuzzy soft sets over UP-algebras. We introduce the notions of the hesitant union and the hesitant intersection of any hesitant fuzzy set, and examine some results on UPalgebras. Afterward, we introduce the notions of the intersection, the union, the restricted intersection, the extended intersection, the AND and the OR of any hesitant fuzzy soft set, and investigate some results over UP-algebras.

\section{Basic results on UP-algebras}

Before we begin our study, we will give the definition of a UP-algebra.

Definition $2.1([5])$. An algebra $A=(A, \cdot, 0)$ of type $(2,0)$ is called a UP-algebra, where $A$ is a nonempty set, - is a binary operation on $A$, and 0 is a fixed element of $A$ (i.e., a nullary operation) if it satisfies the following axioms:

$(\mathrm{UP}-1)(\forall x, y, z \in A)((y \cdot z) \cdot((x \cdot y) \cdot(x \cdot z))=0)$;

$(\mathrm{UP}-2)(\forall x \in A)(0 \cdot x=x)$;

(UP-3) $(\forall x \in A)(x \cdot 0=0)$; and

$(\mathrm{UP}-4)(\forall x, y \in A)(x \cdot y=0, y \cdot x=0 \Rightarrow x=y)$.

From [5], we know that the notion of UP-algebras is a generalization of KU-algebras.

Example 2.2 ([24]). Let $X$ be a universal set and let $\Omega \in \mathcal{P}(X)$. Let $\mathcal{P}_{\Omega}(X)=\{A \in \mathcal{P}(X) \mid \Omega \subseteq A\}$. Define a binary operation $\cdot$ on $\mathcal{P}_{\Omega}(X)$ by putting $A \cdot B=B \cap\left(A^{\prime} \cup \Omega\right)$ for all $A, B \in \mathcal{P}_{\Omega}(X)$. Then $\left(\mathcal{P}_{\Omega}(X), \cdot, \Omega\right)$ is a UP-algebra and we shall call it the generalized power UP-algebra of type 1 with respect to $\Omega$.

In particular, $\left(\mathcal{P}_{\emptyset}(X), \cdot \emptyset\right)$ is the power UP-algebra of type 1 . 
Example 2.3 ([24]). Let $X$ be a universal set and let $\Omega \in \mathcal{P}(X)$. Let $\mathcal{P}^{\Omega}(X)=\{A \in \mathcal{P}(X) \mid A \subseteq \Omega\}$. Define a binary operation $*$ on $\mathcal{P}^{\Omega}(X)$ by putting $A * B=B \cup\left(A^{\prime} \cap \Omega\right)$ for all $A, B \in \mathcal{P}^{\Omega}(X)$. Then $(\mathcal{P} \Omega(X), *, \Omega)$ is a UP-algebra and we shall call it the generalized power UP-algebra of type 2 with respect to $\Omega$.

In particular, $\left(\mathcal{P}^{X}(X), *, X\right)$ is the power UP-algebra of type 2 .

For more examples of UP-algebras, see [2, 6, 23, 24].

In what follows, let $A$ denote UP-algebras unless otherwise specified. The following proposition is very important for the study of UP-algebras.

Proposition $2.4([5,6])$. In a UP-algebra A, the following properties hold:

(1) $(\forall x \in A)(x \cdot x=0)$;

(2) $(\forall x, y, z \in A)(x \cdot y=0, y \cdot z=0 \Rightarrow x \cdot z=0)$;

(3) $(\forall x, y, z \in A)(x \cdot y=0 \Rightarrow(z \cdot x) \cdot(z \cdot y)=0)$;

(4) $(\forall x, y, z \in A)(x \cdot y=0 \Rightarrow(y \cdot z) \cdot(x \cdot z)=0)$;

(5) $(\forall x, y \in A)(x \cdot(y \cdot x)=0)$;

(6) $(\forall x, y \in A)((y \cdot x) \cdot x=0 \Leftrightarrow x=y \cdot x)$;

(7) $(\forall x, y \in A)(x \cdot(y \cdot y)=0)$;

(8) $(\forall a, x, y, z \in A)((x \cdot(y \cdot z)) \cdot(x \cdot((a \cdot y) \cdot(a \cdot z)))=0)$;

(9) $(\forall a, x, y, z \in A)((((a \cdot x) \cdot(a \cdot y)) \cdot z) \cdot((x \cdot y) \cdot z)=0)$;

(10) $(\forall x, y, z \in A)(((x \cdot y) \cdot z) \cdot(y \cdot z)=0)$;

(11) $(\forall x, y, z \in A)(x \cdot y=0 \Rightarrow x \cdot(z \cdot y)=0)$;

(12) $(\forall x, y, z \in A)(((x \cdot y) \cdot z) \cdot(x \cdot(y \cdot z))=0)$; and

(13) $(\forall a, x, y, z \in A)(((x \cdot y) \cdot z) \cdot(y \cdot(a \cdot z))=0)$.

On a UP-algebra $A=(A, \cdot, 0)$, we define a binary relation $\leqslant$ on $A[5]$ as follows:

$$
(\forall x, y \in A)(x \leqslant y \Leftrightarrow x \cdot y=0) .
$$

Definition $2.5([4,5,28])$. A nonempty subset $S$ of a UP-algebra $(A, \cdot, 0)$ is called

(1) a UP-subalgebra of $A$ if $(\forall x, y \in S)(x \cdot y \in S)$;

(2) a UP-filter of $A$ if

(i) the constant 0 of $A$ is in $S$; and

(ii) $(\forall x, y \in A)(x \cdot y \in S, x \in S \Rightarrow y \in S)$;

(3) a UP-ideal of $A$ if

(i) the constant 0 of $A$ is in $S$; and

(ii) $(\forall x, y, z \in A)(x \cdot(y \cdot z) \in S, y \in S \Rightarrow x \cdot z \in S)$;

(4) a strongly UP-ideal of $A$ if

(i) the constant 0 of $A$ is in $S$; and 
(ii) $(\forall x, y, z \in A)((z \cdot y) \cdot(z \cdot x) \in S, y \in S \Rightarrow x \in S)$.

Guntasow et al. [4] proved the generalization that the notion of UP-subalgebras is a generalization of UP-filters, the notion of UP-filters is a generalization of UP-ideals, and the notion of UP-ideals is a generalization of strongly UP-ideals. Moreover, they also proved that a UP-algebra $A$ is the only one strongly UP-ideal of itself.

\section{Basic Results on Hesitant Fuzzy (Soft) Sets}

Definition 3.1 ([32]). Let $X$ be a reference set. A hesitant fuzzy set on $X$ is defined in term of a function $h_{H}$ that when applied to $X$ return a subset of $[0,1]$, that is, $h_{H}: X \rightarrow \mathcal{P}([0,1])$. A hesitant fuzzy set $h_{H}$ can also be viewed as the following mathematical representation:

$$
\mathrm{H}:=\left\{\left(x, \mathrm{~h}_{\mathrm{H}}(x)\right) \mid x \in \mathrm{X}\right\},
$$

where $h_{H}(x)$ is a set of some values in $[0,1]$, denoting the possible membership degrees of the elements $x \in X$ to the set $H$.

Definition 3.2 ([19]). A hesitant fuzzy set $\mathrm{H}$ on $\mathrm{A}$ is called

(1) a hesitant fuzzy UP-subalgebra of $A$ if $(\forall x, y \in A)\left(h_{H}(x \cdot y) \supseteq h_{H}(x) \cap h_{H}(y)\right)$;

(2) a hesitant fuzzy UP-filter of $A$ if

(i) $(\forall x \in A)\left(h_{H}(0) \supseteq h_{H}(x)\right)$; and

(ii) $(\forall x, y \in A)\left(h_{H}(y) \supseteq h_{H}(x \cdot y) \cap h_{H}(x)\right)$;

(3) a hesitant fuzzy UP-ideal of $A$ if

(i) $(\forall x \in A)\left(h_{H}(0) \supseteq h_{H}(x)\right)$; and

(ii) $(\forall x, y, z \in A)\left(h_{H}(x \cdot z) \supseteq h_{H}(x \cdot(y \cdot z)) \cap h_{H}(y)\right)$;

(4) a hesitant fuzzy strongly UP-ideal of $A$ if

(i) $(\forall x \in A)\left(h_{H}(0) \supseteq h_{H}(x)\right)$; and

(ii) $(\forall x, y, z \in A)\left(h_{H}(x) \supseteq h_{H}((z \cdot y) \cdot(z \cdot x)) \cap h_{H}(y)\right)$.

Mosrijai et al. [19] proved that the notion of hesitant fuzzy UP-subalgebras of UP-algebras is a generalization of hesitant fuzzy UP-filters, the notion of hesitant fuzzy UP-filters of UP-algebras is a generalization of hesitant fuzzy UP-ideals, and the notion of hesitant fuzzy UP-ideals of UP-algebras is a generalization of hesitant fuzzy strongly UP-ideals.

Theorem 3.3 ([19]). A hesitant fuzzy set $\mathrm{H}$ on $\mathrm{A}$ is a hesitant fuzzy strongly UP-ideal of $\mathrm{A}$ if and only if it is a constant hesitant fuzzy set on $A$.

Definition 3.4 ([15]). A hesitant fuzzy set $\mathrm{H}$ on $\mathrm{A}$ is called

(1) an anti-hesitant fuzzy UP-subalgebra of $A$ if $(\forall x, y \in A)\left(h_{H}(x \cdot y) \subseteq h_{H}(x) \cup h_{H}(y)\right)$;

(2) an anti-hesitant fuzzy UP-filter of $A$ if

(i) $(\forall x \in A)\left(h_{H}(0) \subseteq h_{H}(x)\right)$; and

(ii) $(\forall x, y \in A)\left(h_{H}(y) \subseteq h_{H}(x \cdot y) \cup h_{H}(x)\right)$;

(3) an anti-hesitant fuzzy UP-ideal of A if 
(i) $(\forall x \in A)\left(h_{H}(0) \subseteq h_{H}(x)\right)$; and

(ii) $(\forall x, y, z \in A)\left(h_{H}(x \cdot z) \subseteq h_{H}(x \cdot(y \cdot z)) \cup h_{H}(y)\right)$;

(4) an anti-hesitant fuzzy strongly UP-ideal of $A$ if

(i) $(\forall x \in A)\left(\mathrm{h}_{\mathrm{H}}(0) \subseteq \mathrm{h}_{\mathrm{H}}(\mathrm{x})\right)$; and

(ii) $(\forall x, y, z \in A)\left(h_{H}(x) \subseteq h_{H}((z \cdot y) \cdot(z \cdot x)) \cup h_{H}(y)\right)$.

Mosrijai and Iampan [15] proved that the notion of anti-hesitant fuzzy UP-subalgebras of UP-algebras is a generalization of anti-hesitant fuzzy UP-filters, the notion of anti-hesitant fuzzy UP-filters of UPalgebras is a generalization of anti-hesitant fuzzy UP-ideals, and the notion of anti-hesitant fuzzy UPideals of UP-algebras is a generalization of anti-hesitant fuzzy strongly UP-ideals.

Theorem 3.5 ([15]). A hesitant fuzzy set $\mathrm{H}$ on $\mathrm{A}$ is an anti-hesitant fuzzy strongly UP-ideal of $\mathrm{A}$ if and only if it is a constant hesitant fuzzy set on A.

Definition 3.6 ([3]). Let $X$ be a reference set (or an initial universe set) and $P$ be a set of parameters. Let $\operatorname{HFS}(X)$ be the set of all hesitant fuzzy sets on $X$ and $Y$ be a nonempty subset of $P$. A pair $(\widetilde{H}, Y)$ is called a hesitant fuzzy soft set over $\mathrm{X}$, where $\widetilde{\mathrm{H}}$ is a mapping given by

$$
\widetilde{\mathrm{H}}: \mathrm{Y} \rightarrow \mathrm{HFS}(X), p \mapsto \widetilde{\mathrm{H}}[\mathrm{p}] .
$$

Definition 3.7 ([16]). Let $Y$ be a nonempty subset of P. A hesitant fuzzy soft set $(\widetilde{H}, Y)$ over $A$ is called a hesitant fuzzy soft UP-subalgebra (resp., hesitant fuzzy soft UP-filter, hesitant fuzzy soft UP-ideal, hesitant fuzzy soft strongly UP-ideal) based on $p \in Y$ (we shortly call a $p$-hesitant fuzzy soft UP-subalgebra (resp., p-hesitant fuzzy soft UP-filter, $\mathrm{p}$-hesitant fuzzy soft UP-ideal, $\mathrm{p}$-hesitant fuzzy soft strongly UP-ideal)) of $A$ if the hesitant fuzzy set

$$
\widetilde{\mathrm{H}}[\mathrm{p}]:=\left\{\left(\mathrm{a}, \mathrm{h}_{\widetilde{\mathrm{H}}[\mathrm{p}]}(\mathrm{a})\right) \mid \mathrm{a} \in \mathrm{A}\right\}
$$

on $A$ is a hesitant fuzzy UP-subalgebra (resp., hesitant fuzzy UP-filter, hesitant fuzzy UP-ideal, hesitant fuzzy strongly UP-ideal) of $A$. If $(\widetilde{H}, Y)$ is a p-hesitant fuzzy soft UP-subalgebra (resp., hesitant fuzzy soft UP-filter, hesitant fuzzy soft UP-ideal, hesitant fuzzy soft strongly UP-ideal) of $A$ for all $p \in Y$, we state that $(\widetilde{\mathrm{H}}, \mathrm{Y})$ is a hesitant fuzzy soft UP-subalgebra (resp., hesitant fuzzy soft UP-filter, hesitant fuzzy soft UP-ideal, hesitant fuzzy soft strongly UP-ideal) of A.

Definition 3.8 ([18]). Let $Y$ be a nonempty subset of $P$. A hesitant fuzzy soft set $(\widetilde{H}, Y)$ over $A$ is called an anti-hesitant fuzzy soft UP-subalgebra (resp., anti-hesitant fuzzy soft UP-filter, anti-hesitant fuzzy soft UP-ideal, anti-hesitant fuzzy soft strongly UP-ideal) based on $p \in Y$ (we shortly call a p-anti-hesitant fuzzy soft UPsubalgebra (resp., p-anti-hesitant fuzzy soft UP-filter, p-anti-hesitant fuzzy soft UP-ideal, p-anti-hesitant fuzzy soft strongly UP-ideal)) of $A$ if the hesitant fuzzy set

$$
\widetilde{\mathrm{H}}[\mathrm{p}]:=\left\{\left(\mathrm{a}, \mathrm{h}_{\widetilde{\mathrm{H}}[\mathrm{p}]}(\mathrm{a})\right) \mid \mathrm{a} \in A\right\}
$$

on $A$ is an anti-hesitant fuzzy UP-subalgebra of $A$. If $(\widetilde{H}, Y)$ is a p-anti-hesitant fuzzy soft UP-subalgebra of $A$ for all $p \in Y$, we state that $(\widetilde{H}, Y)$ is an anti-hesitant fuzzy soft UP-subalgebra (resp., anti-hesitant fuzzy soft UP-filter, anti-hesitant fuzzy soft UP-ideal, anti-hesitant fuzzy soft strongly UP-ideal) of A.

\section{Operations on hesitant fuzzy sets}

Torra [32] defined many operations on hesitant fuzzy sets in 2010. For instance, the intersection operation $\cap_{\mathrm{T}}$, the union operation $\cup_{\mathrm{T}}$ and so on. 
Definition 4.1 ([32]). Let $\mathrm{H}$ and $\mathrm{F}$ be hesitant fuzzy sets on a reference set $\mathrm{X}$. Then the following operations are defined:

(1) $\mathrm{H} \cap_{\mathrm{T}} \mathrm{F}=\left\{\left(x,\left\{h \in\left(h_{\mathrm{H}}(x) \cup h_{\mathrm{F}}(x)\right) \mid h \leqslant \min \left\{\operatorname{suph}_{\mathrm{H}}(x), \sup _{\mathrm{F}}(\mathrm{x})\right\}\right\}\right) \mid x \in X\right\}$, and

(2) $\mathrm{H} \cup_{\mathrm{T}} \mathrm{F}=\left\{\left(x,\left\{h \in\left(h_{\mathrm{H}}(x) \cup h_{\mathrm{F}}(x)\right) \mid h \geqslant \max \left\{\inf h_{\mathrm{H}}(x), \inf h_{\mathrm{F}}(x)\right\}\right\}\right) \mid x \in X\right\}$.

We can write the intersection and the union of hesitant fuzzy sets with different form by

$$
\mathrm{H} \cap_{\mathrm{T}} \mathrm{F}=\left\{\left(x, \bigcup_{\gamma_{1} \in \mathrm{h}_{\mathrm{H}}(\mathrm{x}), \gamma_{2} \in \mathrm{h}_{\mathrm{F}}(x)}\left\{\min \left\{\gamma_{1}, \gamma_{2}\right\}\right\}\right) \mid x \in \mathrm{X}\right\}
$$

and

$$
\mathrm{H} \cup_{\mathrm{T}} \mathrm{F}=\left\{\left(x, \bigcup_{\gamma_{1} \in \mathrm{h}_{\mathrm{H}}(\mathrm{x}), \gamma_{2} \in \mathrm{h}_{\mathrm{F}}(\mathrm{x})}\left\{\max \left\{\gamma_{1}, \gamma_{2}\right\}\right\}\right) \mid x \in \mathrm{X}\right\} .
$$

First, we will consider about results of the intersection and the union of hesitant fuzzy sets on UPalgebras.

The following example show that the intersection of hesitant fuzzy UP-subalgebras of $A$ is not a hesitant fuzzy UP-subalgebra of $A$.

Example 4.2. Let $A=\{0,1,2,3\}$ be a set with a binary operation - defined by the following Cayley table:

\begin{tabular}{l|llll}
$\cdot$ & 0 & 1 & 2 & 3 \\
\hline 0 & 0 & 1 & 2 & 3 \\
1 & 0 & 0 & 2 & 2 \\
2 & 0 & 1 & 0 & 1 \\
3 & 0 & 0 & 0 & 0
\end{tabular}

Then $(A, \cdot, 0)$ is a UP-algebra. We define two hesitant fuzzy sets $\mathrm{H}_{1}$ and $\mathrm{H}_{2}$ on $A$ as follows:

$$
\mathrm{h}_{\mathrm{H}_{1}}(x)=\left\{\begin{array}{ll}
\{0,0.5,1\}, & \text { if } x=0, \\
\{0\}, & \text { if } x=1, \\
\{0.5\}, & \text { if } x=2, \\
\{1\}, & \text { if } x=3,
\end{array} \text {, } \quad \text { and } \mathrm{h}_{\mathrm{H}_{2}}(x)= \begin{cases}\{0.5,1\}, & \text { if } x=0, \\
\{0.5\}, & \text { if } x=1, \\
\{0,5\}, & \text { if } x=2, \\
\{0.5,1\}, & \text { if } x=3 .\end{cases}\right.
$$

Then $\mathrm{H}_{1}$ and $\mathrm{H}_{2}$ are hesitant fuzzy UP-subalgebras of $A$. We thus obtain the intersection of $\mathrm{H}_{1}$ and $\mathrm{H}_{2}$ as follows:

$$
\mathrm{H}_{1} \cap_{\mathrm{T}} \mathrm{H}_{2}(x)= \begin{cases}\{0,0.5,1\}, & \text { if } x=0, \\ \{0\}, & \text { if } x=1, \\ \{0.5\}, & \text { if } x=2, \\ \{0.5,1\}, & \text { if } x=3\end{cases}
$$

Therefore, $\mathrm{H}_{1} \cap_{T} \mathrm{H}_{2}$ is not a hesitant fuzzy UP-subalgebra of $A$ because

$$
\mathrm{h}_{\mathrm{H}_{1} \cap_{\mathrm{T}} \mathrm{H}_{2}}(2 \cdot 3)=\mathrm{h}_{\mathrm{H}_{1} \cap \cap_{\mathrm{T}} \mathrm{H}_{2}}(1)=\{0\} \nsupseteq\{0.5\}=\{0.5\} \cap\{0.5,1\}=\mathrm{h}_{\mathrm{H}_{1} \cap_{\mathrm{T}} \mathrm{H}_{2}}(2) \cap \mathrm{h}_{\mathrm{H}_{1} \cap_{\mathrm{T}} \mathrm{H}_{2}}(3) .
$$

The following example shows that the intersection of hesitant fuzzy UP-filters of $A$ is not a hesitant fuzzy UP-filter of $A$.

Example 4.3. Let $A=\{0,1,2,3\}$ be a set with a binary operation - defined by the following Cayley table:

$$
\begin{array}{c|llll}
\cdot & 0 & 1 & 2 & 3 \\
\hline 0 & 0 & 1 & 2 & 3 \\
1 & 0 & 0 & 3 & 3 \\
2 & 0 & 1 & 0 & 0 \\
3 & 0 & 1 & 2 & 0
\end{array}
$$


Then $(A, \cdot, 0)$ is a UP-algebra. We define two hesitant fuzzy sets $\mathrm{H}_{1}$ and $\mathrm{H}_{2}$ on $A$ as follows:

$$
\mathrm{h}_{\mathrm{H}_{1}}(x)=\left\{\begin{array}{ll}
\{0,1\}, & \text { if } x=0, \\
\{1\}, & \text { if } x=1, \\
\emptyset, & \text { if } x=2, \\
\{0\}, & \text { if } x=3,
\end{array} \text {, } \quad \text { and } \mathrm{h}_{\mathrm{H}_{2}}(x)= \begin{cases}\{0,0.5\}, & \text { if } x=0, \\
\{0,0.5\}, & \text { if } x=1, \\
\{0,5\}, & \text { if } x=2, \\
\{0.5\}, & \text { if } x=3\end{cases}\right.
$$

Then $\mathrm{H}_{1}$ and $\mathrm{H}_{2}$ are hesitant fuzzy UP-filters of $\mathrm{A}$. We thus obtain the intersection of $\mathrm{H}_{1}$ and $\mathrm{H}_{2}$ as follows:

$$
\mathrm{H}_{1} \cap_{\mathrm{T}} \mathrm{H}_{2}(x)= \begin{cases}\{0,0.5\}, & \text { if } x=0, \\ \{0,0.5\}, & \text { if } x=1, \\ \{0.5\}, & \text { if } x=2, \\ \{0\}, & \text { if } x=3 .\end{cases}
$$

Therefore, $\mathrm{H}_{1} \cap_{T} \mathrm{H}_{2}$ is not a hesitant fuzzy UP-filter of $A$ because

$$
\mathrm{h}_{\mathrm{H}_{1} \cap_{\mathrm{T}} \mathrm{H}_{2}}(3)=\{0\} \nsupseteq\{0.5\}=\{0,0.5\} \cap\{0.5\}=\mathrm{h}_{\mathrm{H}_{1} \cap_{\mathrm{T}} \mathrm{H}_{2}}(0) \cap \mathrm{h}_{\mathrm{H}_{1} \cap_{\mathrm{T}} \mathrm{H}_{2}}(2)=\mathrm{h}_{\mathrm{H}_{1} \cap_{\mathrm{T}} \mathrm{H}_{2}}(2 \cdot 3) \cap \mathrm{h}_{\mathrm{H}_{1} \cap_{\mathrm{T}} \mathrm{H}_{2}}(2) .
$$

The following example shows that the intersection of hesitant fuzzy UP-ideals of $A$ is not a hesitant fuzzy UP-ideal of $A$.

Example 4.4. Let $A=\{0,1,2,3\}$ be a set with a binary operation - defined by the following Cayley table:

\begin{tabular}{l|llll}
$\cdot$ & 0 & 1 & 2 & 3 \\
\hline 0 & 0 & 1 & 2 & 3 \\
1 & 0 & 0 & 2 & 2 \\
2 & 0 & 1 & 0 & 1 \\
3 & 0 & 0 & 0 & 0
\end{tabular}

Then $(A, \cdot, 0)$ is a UP-algebra. We define two hesitant fuzzy sets $H_{1}$ and $H_{2}$ on $A$ as follows:

$$
\mathrm{h}_{\mathrm{H}_{1}}(x)=\left\{\begin{array}{ll}
\{0,0.1\} & \text { if } x=0, \\
\{0,0.1\} & \text { if } x=1, \\
\{0.1\} & \text { if } x=2, \\
\{0.1\} & \text { if } x=3,
\end{array} \text {, and } \mathrm{h}_{\mathrm{H}_{2}}(x)= \begin{cases}\{0,0.1\} & \text { if } x=0, \\
\{0.1\} & \text { if } x=1, \\
\{0,0,1\} & \text { if } x=2, \\
\{0.1\} & \text { if } x=3\end{cases}\right.
$$

Then $\mathrm{H}_{1}$ and $\mathrm{H}_{2}$ are hesitant fuzzy UP-ideals of $A$. We thus obtain the intersection of $\mathrm{H}_{1}$ and $\mathrm{H}_{2}$ as follows:

$$
\mathrm{H}_{1} \cap_{\mathrm{T}} \mathrm{H}_{2}(x)= \begin{cases}\{0,0.1\}, & \text { if } x=0, \\ \{0,0.1\}, & \text { if } x=1, \\ \{0,0.1\}, & \text { if } x=2, \\ \{0.1\}, & \text { if } x=3\end{cases}
$$

Therefore, $\mathrm{H}_{1} \cap_{\mathrm{T}} \mathrm{H}_{2}$ is not a hesitant fuzzy UP-ideal of $A$ because

$$
\begin{aligned}
\mathrm{h}_{\mathrm{H}_{1} \cap_{\mathrm{T}} \mathrm{H}_{2}}(0 \cdot 3)=\mathrm{h}_{\mathrm{H}_{1} \cap_{\mathrm{T}} \mathrm{H}_{2}}(3)=\{0.1\} \nsupseteq\{0,0.1\}=\{0,0.1\} \cap\{0,0.1\} & =\mathrm{h}_{\mathrm{H}_{1} \cap_{\mathrm{T}} \mathrm{H}_{2}}(2) \cap \mathrm{h}_{\mathrm{H}_{1} \cap_{\mathrm{T}} \mathrm{H}_{2}}(2) \\
& =\mathrm{h}_{\mathrm{H}_{1} \cap_{\mathrm{T}} \mathrm{H}_{2}}(0 \cdot(1 \cdot 3)) \cap \mathrm{h}_{\mathrm{H}_{1} \cap_{\mathrm{T}} \mathrm{H}_{2}}(1) .
\end{aligned}
$$

Theorem 4.5. The intersection of hesitant fuzzy strongly UP-ideals of $\mathrm{A}$ is a hesitant fuzzy strongly UP-ideals of A.

Proof. Assume that $\mathrm{H}$ and $\mathrm{G}$ are hesitant fuzzy strongly UP-ideals of $A$. By Theorem 3.3, we have $\mathrm{H}$ and $G$ are constant hesitant fuzzy sets of $A$. Thus $H \cap_{T} G$ is a constant hesitant fuzzy set of $A$. By Theorem 3.3, we can conclude that $H \cap_{T} \mathrm{G}$ is a hesitant fuzzy strongly UP-ideal of $A$. 
The following example shows that the intersection of anti-hesitant fuzzy UP-subalgebras of $A$ is not an anti-hesitant fuzzy UP-subalgebra of $A$.

Example 4.6. Let $A=\{0,1,2,3\}$ be a set with a binary operation - defined by the following Cayley table:

\begin{tabular}{l|llll}
$\cdot$ & 0 & 1 & 2 & 3 \\
\hline 0 & 0 & 1 & 2 & 3 \\
1 & 0 & 0 & 2 & 2 \\
2 & 0 & 1 & 0 & 1 \\
3 & 0 & 0 & 0 & 0
\end{tabular}

Then $(A, \cdot, 0)$ is a UP-algebra. We define two hesitant fuzzy sets $H_{1}$ and $H_{2}$ on $A$ as follows:

$$
h_{\mathrm{H}_{1}}(x)=\left\{\begin{array}{ll}
\{0.7\}, & \text { if } x=0, \\
{[0.7,0.8),} & \text { if } x=1, \\
{[0.7,0.9],} & \text { if } x=2, \\
{[0.6,0.9],} & \text { if } x=3,
\end{array} \text {. } \quad \text { and } h_{\mathrm{H}_{2}}(x)= \begin{cases}\{0.7\}, & \text { if } x=0, \\
\{0.7,0.8\}, & \text { if } x=1, \\
\{0.7,0.8\}, & \text { if } x=2, \\
\{0.7\}, & \text { if } x=3 .\end{cases}\right.
$$

Then $\mathrm{H}_{1}$ and $\mathrm{H}_{2}$ are anti-hesitant fuzzy UP-subalgebras of $A$. We thus obtain the intersection of $\mathrm{H}_{1}$ and $\mathrm{H}_{2}$ as follows:

$$
\mathrm{H}_{1} \cap_{\mathrm{T}} \mathrm{H}_{2}(x)= \begin{cases}\{0.7\}, & \text { if } x=0, \\ {[0.7,0.8),} & \text { if } x=1, \\ {[0.7,0.9],} & \text { if } x=2, \\ {[0.6,0.7],} & \text { if } x=3\end{cases}
$$

Therefore, $\mathrm{H}_{1} \cap_{\mathrm{T}} \mathrm{H}_{2}$ is not an anti-hesitant fuzzy UP-subalgebra of $A$ because

$$
\mathrm{h}_{\mathrm{H}_{1} \cap \cap_{\mathrm{T}} \mathrm{H}_{2}}(1 \cdot 3)=\mathrm{h}_{\mathrm{H}_{1} \cap \cap_{\mathrm{T}} \mathrm{H}_{2}}(2)=[0.7,0.9] \nsubseteq[0.6,0.8)=[0.7,0.8) \cup[0.6,0.7]=\mathrm{h}_{\mathrm{H}_{1} \cap_{\mathrm{T}} \mathrm{H}_{2}}(1) \cup \mathrm{h}_{\mathrm{H}_{1} \cap_{\mathrm{T}} \mathrm{H}_{2}}(3) .
$$

The following example shows that the intersection of anti-hesitant fuzzy UP-filters of $A$ is not an anti-hesitant fuzzy UP-filter of $A$.

Example 4.7. Let $A=\{0,1,2,3\}$ be a set with a binary operation - defined by the following Cayley table:

$$
\begin{array}{l|llll}
\cdot & 0 & 1 & 2 & 3 \\
\hline 0 & 0 & 1 & 2 & 3 \\
1 & 0 & 0 & 3 & 3 \\
2 & 0 & 1 & 0 & 0 \\
3 & 0 & 1 & 2 & 0
\end{array}
$$

Then $(A, \cdot, 0)$ is a UP-algebra. We define two hesitant fuzzy sets $\mathrm{H}_{1}$ and $\mathrm{H}_{2}$ on $A$ as follows:

$$
h_{\mathrm{H}_{1}}(x)=\left\{\begin{array}{ll}
\emptyset, & \text { if } x=0, \\
\{1\}, & \text { if } x=1, \\
\{0\}, & \text { if } x=2, \\
\{0\}, & \text { if } x=3,
\end{array} \text {, and } h_{\mathrm{H}_{2}}(x)= \begin{cases}\{0.2\}, & \text { if } x=0, \\
\{0,0.2\}, & \text { if } x=1, \\
\{0.2,0.8,1\}, & \text { if } x=2, \\
\{0.2,1\}, & \text { if } x=3\end{cases}\right.
$$

Then $\mathrm{H}_{1}$ and $\mathrm{H}_{2}$ are anti-hesitant fuzzy UP-filters of $A$. We thus obtain the intersection of $\mathrm{H}_{1}$ and $\mathrm{H}_{2}$ as follows:

$$
\mathrm{H}_{1} \cap_{\mathrm{T}} \mathrm{H}_{2}(x)= \begin{cases}\{0.2\}, & \text { if } x=0, \\ \{0,0.2\}, & \text { if } x=1, \\ \{0\}, & \text { if } x=2, \\ \{0\}, & \text { if } x=3 .\end{cases}
$$

Therefore, $\mathrm{H}_{1} \cap_{\mathrm{T}} \mathrm{H}_{2}$ is not an anti-hesitant fuzzy UP-filter of $A$ because

$$
\mathrm{h}_{\mathrm{H}_{1} \cap_{\mathrm{T}} \mathrm{H}_{2}}(0)=\{0.2\} \nsubseteq\{0\}=\mathrm{h}_{\mathrm{H}_{1} \cap_{\mathrm{T}} \mathrm{H}_{2}}(2) .
$$


The following example shows that the intersection of anti-hesitant fuzzy UP-ideals of $A$ is not an anti-hesitant fuzzy UP-ideal of $A$.

Example 4.8. Let $A=\{0,1,2,3\}$ be a set with a binary operation - defined by the following Cayley table:

\begin{tabular}{l|llll}
$\cdot$ & 0 & 1 & 2 & 3 \\
\hline 0 & 0 & 1 & 2 & 3 \\
1 & 0 & 0 & 2 & 2 \\
2 & 0 & 1 & 0 & 1 \\
3 & 0 & 0 & 2 & 0
\end{tabular}

Then $(A, \cdot, 0)$ is a UP-algebra. We define two hesitant fuzzy sets $H_{1}$ and $H_{2}$ on $A$ as follows:

$$
\mathrm{h}_{\mathrm{H}_{1}}(x)=\left\{\begin{array}{ll}
\{1\}, & \text { if } x=0, \\
\{1\}, & \text { if } x=1, \\
\{0.8,1\}, & \text { if } x=2, \\
\{0.8,1\}, & \text { if } x=3,
\end{array}\right. \text {. }
$$

Then $\mathrm{H}_{1}$ and $\mathrm{H}_{2}$ are anti-hesitant fuzzy UP-ideals of $A$. We thus obtain the intersection of $\mathrm{H}_{1}$ and $\mathrm{H}_{2}$ as follows:

$$
\mathrm{H}_{1} \cap_{\mathrm{T}} \mathrm{H}_{2}(x)= \begin{cases}\{1\}, & \text { if } x=0 \\ \{0.5\}, & \text { if } x=1, \\ \{0.8,1\}, & \text { if } x=2 \\ \{0.5\}, & \text { if } x=3\end{cases}
$$

Therefore, $\mathrm{H}_{1} \cap_{T} \mathrm{H}_{2}$ is not an anti-hesitant fuzzy UP-ideal of $A$ because

$$
\mathrm{h}_{\mathrm{H}_{1} \cap_{\mathrm{T}} \mathrm{H}_{2}}(0)=\{1\} \nsubseteq\{0.5\}=\mathrm{h}_{\mathrm{H}_{1} \cap_{\mathrm{T}} \mathrm{H}_{2}}(1) .
$$

Theorem 4.9. The intersection of anti-hesitant fuzzy strongly UP-ideals of $\mathrm{A}$ is an anti-hesitant fuzzy strongly UP-ideals of A.

Proof. Assume that $\mathrm{H}$ and $\mathrm{G}$ are anti-hesitant fuzzy strongly UP-ideals of $A$. By Theorem 3.5, we have $H$ and $G$ are constant hesitant fuzzy sets of $A$. Thus $H \cap_{T} G$ is a constant hesitant fuzzy set of $A$. By Theorem 3.5, we can conclude that $\mathrm{H} \cap_{\mathrm{T}} \mathrm{G}$ is an anti-hesitant fuzzy strongly UP-ideal of $A$.

The following example shows that the union of hesitant fuzzy UP-subalgebras of $A$ is not a hesitant fuzzy UP-subalgebra of A.

Example 4.10. Let $A=\{0,1,2,3\}$ be a set with a binary operation - defined by the following Cayley table:

$$
\begin{array}{l|llll}
\cdot & 0 & 1 & 2 & 3 \\
\hline 0 & 0 & 1 & 2 & 3 \\
1 & 0 & 0 & 2 & 2 \\
2 & 0 & 1 & 0 & 1 \\
3 & 0 & 0 & 0 & 0
\end{array}
$$

Then $(A, \cdot, 0)$ is a UP-algebra. We define two hesitant fuzzy sets $\mathrm{H}_{1}$ and $\mathrm{H}_{2}$ on $A$ as follows:

$$
\mathrm{h}_{\mathrm{H}_{1}}(x)=\left\{\begin{array}{ll}
\{0,0.5,1\}, & \text { if } x=0, \\
\{1\}, & \text { if } x=1, \\
\{0.5\}, & \text { if } x=2, \\
\{0\}, & \text { if } x=3,
\end{array} \text {, } \quad \text { and } \mathrm{h}_{\mathrm{H}_{2}}(x)= \begin{cases}\{0,0.5\}, & \text { if } x=0, \\
\{0.5\}, & \text { if } x=1, \\
\{0,5\}, & \text { if } x=2, \\
\{0,0.5\}, & \text { if } x=3 .\end{cases}\right.
$$


Then $\mathrm{H}_{1}$ and $\mathrm{H}_{2}$ are hesitant fuzzy UP-subalgebras of $A$. We thus obtain the intersection of $\mathrm{H}_{1}$ and $\mathrm{H}_{2}$ as follows:

$$
\mathrm{H}_{1} \cup_{\mathrm{T}} \mathrm{H}_{2}(x)= \begin{cases}\{0,0.5,1\}, & \text { if } x=0, \\ \{1\}, & \text { if } x=1, \\ \{0.5\}, & \text { if } x=2, \\ \{0,0.5\}, & \text { if } x=3\end{cases}
$$

Therefore, $\mathrm{H}_{1} \cup_{\mathrm{T}} \mathrm{H}_{2}$ is not a hesitant fuzzy UP-subalgebra of $A$ because

$$
\mathrm{h}_{\mathrm{H}_{1} \cup_{\mathrm{T}} \mathrm{H}_{2}}(2 \cdot 3)=\mathrm{h}_{\mathrm{H}_{1} \cup_{\mathrm{T}} \mathrm{H}_{2}}(1)=\{1\} \nsupseteq\{0.5\}=\{0.5\} \cap\{0,0.5\}=\mathrm{h}_{\mathrm{H}_{1} \cup_{\mathrm{T}} \mathrm{H}_{2}}(2) \cap \mathrm{h}_{\mathrm{H}_{1} \cup_{\mathrm{T}} \mathrm{H}_{2}}(3) .
$$

The following example shows that the union of hesitant fuzzy UP-filters of $A$ is not a hesitant fuzzy UP-filter of $A$.

Example 4.11. Let $A=\{0,1,2,3\}$ be a set with a binary operation - defined by the following Cayley table:

\begin{tabular}{l|llll}
$\cdot$ & 0 & 1 & 2 & 3 \\
\hline 0 & 0 & 1 & 2 & 3 \\
1 & 0 & 0 & 3 & 3 \\
2 & 0 & 1 & 0 & 0 \\
3 & 0 & 1 & 2 & 0
\end{tabular}

Then $(A, \cdot, 0)$ is a UP-algebra. We define two hesitant fuzzy sets $\mathrm{H}_{1}$ and $\mathrm{H}_{2}$ on $A$ as follows:

$$
\mathrm{h}_{\mathrm{H}_{1}}(x)=\left\{\begin{array}{ll}
\{0,1\}, & \text { if } x=0, \\
\{0\}, & \text { if } x=1, \\
\emptyset, & \text { if } x=2, \\
\{1\}, & \text { if } x=3,
\end{array} \text {, nd } \mathrm{h}_{\mathrm{H}_{2}}(x)= \begin{cases}\{0.5,1\}, & \text { if } x=0, \\
\{0.5,1\}, & \text { if } x=1, \\
\{0,5\}, & \text { if } x=2, \\
\{0.5\}, & \text { if } x=3\end{cases}\right.
$$

Then $\mathrm{H}_{1}$ and $\mathrm{H}_{2}$ are hesitant fuzzy UP-filters of $A$. We thus obtain the intersection of $\mathrm{H}_{1}$ and $\mathrm{H}_{2}$ as follows:

$$
\mathrm{H}_{1} \cup_{\mathrm{T}} \mathrm{H}_{2}(x)= \begin{cases}\{0.5,1\}, & \text { if } x=0, \\ \{0.5,1\}, & \text { if } x=1, \\ \{0.5\}, & \text { if } x=2, \\ \{1\}, & \text { if } x=3\end{cases}
$$

Therefore, $\mathrm{H}_{1} \cup_{T} \mathrm{H}_{2}$ is not a hesitant fuzzy UP-filter of $A$ because

$$
\mathrm{h}_{\mathrm{H}_{1} \cup_{T} \mathrm{H}_{2}}(3)=\{1\} \nsupseteq\{0.5\}=\{0.5,1\} \cap\{0.5\}=\mathrm{h}_{\mathrm{H}_{1} \cup_{T} \mathrm{H}_{2}}(0) \cap \mathrm{h}_{\mathrm{H}_{1} \cup_{T} \mathrm{H}_{2}}(2)=\mathrm{h}_{\mathrm{H}_{1} \cup_{T} \mathrm{H}_{2}}(2 \cdot 3) \cap \mathrm{h}_{\mathrm{H}_{1} \cup_{T} \mathrm{H}_{2}}(2) .
$$

The following example shows that the union of hesitant fuzzy UP-ideals of $A$ is not a hesitant fuzzy UP-ideal of $A$.

Example 4.12. Let $A=\{0,1,2,3\}$ be a set with a binary operation - defined by the following Cayley table:

\begin{tabular}{l|llll} 
& 0 & 1 & 2 & 3 \\
\hline 0 & 0 & 1 & 2 & 3 \\
1 & 0 & 0 & 2 & 2 \\
2 & 0 & 1 & 0 & 1 \\
3 & 0 & 0 & 0 & 0
\end{tabular}

Then $(A, \cdot, 0)$ is a UP-algebra. We define two hesitant fuzzy sets $\mathrm{H}_{1}$ and $\mathrm{H}_{2}$ on $A$ as follows:

$$
h_{\mathrm{H}_{1}}(x)=\left\{\begin{array}{ll}
\{0.9,1\}, & \text { if } x=0, \\
\{0.9,1\}, & \text { if } x=1, \\
\{0.9\}, & \text { if } x=2, \\
\{0.9\}, & \text { if } x=3,
\end{array} \text {, } \quad \text { and } h_{\mathrm{H}_{2}}(x)= \begin{cases}\{0.9,1\}, & \text { if } x=0, \\
\{0.9\}, & \text { if } x=1, \\
\{0,9,1\}, & \text { if } x=2, \\
\{0.9\}, & \text { if } x=3 .\end{cases}\right.
$$


Then $\mathrm{H}_{1}$ and $\mathrm{H}_{2}$ are hesitant fuzzy UP-ideals of $A$. We thus obtain the intersection of $\mathrm{H}_{1}$ and $\mathrm{H}_{2}$ as follows:

$$
\mathrm{H}_{1} \cap_{\mathrm{T}} \mathrm{H}_{2}(\mathrm{x})= \begin{cases}\{0.9,1\}, & \text { if } \mathrm{x}=0, \\ \{0.9,1\}, & \text { if } \mathrm{x}=1, \\ \{0.9,1\}, & \text { if } \mathrm{x}=2, \\ \{0.9\}, & \text { if } \mathrm{x}=3 .\end{cases}
$$

Therefore, $\mathrm{H}_{1} \cap_{\mathrm{T}} \mathrm{H}_{2}$ is not a hesitant fuzzy UP-ideal of $A$ because

$$
\begin{aligned}
\mathrm{h}_{\mathrm{H}_{1} \cap_{\mathrm{T}} \mathrm{H}_{2}}(0 \cdot 3)=\mathrm{h}_{\mathrm{H}_{1} \cap_{\mathrm{T}} \mathrm{H}_{2}}(3)=\{0.9\} \nsupseteq\{0.9,1\} & =\{0.9,1\} \cap\{0.9,1\} \\
& =\mathrm{h}_{\mathrm{H}_{1} \cap_{\mathrm{T}} \mathrm{H}_{2}}(2) \cap \mathrm{h}_{\mathrm{H}_{1} \cap_{\mathrm{T}} \mathrm{H}_{2}}(2)=\mathrm{h}_{\mathrm{H}_{1} \cap_{\mathrm{T}} \mathrm{H}_{2}}(0 \cdot(1 \cdot 3)) \cap \mathrm{h}_{\mathrm{H}_{1} \cap_{\mathrm{T}} \mathrm{H}_{2}}(1) .
\end{aligned}
$$

Theorem 4.13. The union of hesitant fuzzy strongly UP-ideals of $A$ is a hesitant fuzzy strongly UP-ideals of A.

Proof. Assume that $\mathrm{H}$ and $\mathrm{G}$ are hesitant fuzzy strongly UP-ideals of $A$. By Theorem 3.3, we have $\mathrm{H}$ and $G$ are constant hesitant fuzzy sets of $A$. Thus $H \cup_{T} G$ is a constant hesitant fuzzy set of $A$. By Theorem 3.3, we can conclude that $H \cup_{T} G$ is a hesitant fuzzy strongly UP-ideal of $A$.

The following example shows that the union of anti-hesitant fuzzy UP-subalgebras of $A$ is not an anti-hesitant fuzzy UP-subalgebra of $A$.

Example 4.14. Let $A=\{0,1,2,3\}$ be a set with a binary operation - defined by the following Cayley table:

\begin{tabular}{l|llll}
$\cdot$ & 0 & 1 & 2 & 3 \\
\hline 0 & 0 & 1 & 2 & 3 \\
1 & 0 & 0 & 2 & 2 \\
2 & 0 & 1 & 0 & 1 \\
3 & 0 & 0 & 0 & 0
\end{tabular}

Then $(A, \cdot, 0)$ is a UP-algebra. We define two hesitant fuzzy sets $\mathrm{H}_{1}$ and $\mathrm{H}_{2}$ on $A$ as follows:

$$
h_{\mathrm{H}_{1}}(x)=\left\{\begin{array}{ll}
\{0.3\}, & \text { if } x=0, \\
{[0.2,0.3],} & \text { if } x=1, \\
{[0.1,0.3],} & \text { if } x=2, \\
{[0.1,0.4],} & \text { if } x=3,
\end{array} \text {, and } h_{\mathrm{H}_{2}}(x)= \begin{cases}\{0.3\}, & \text { if } x=0, \\
\{0.2,0.3\}, & \text { if } x=1, \\
\{0.2,0.3\}, & \text { if } x=2, \\
\{0.3\}, & \text { if } x=3\end{cases}\right.
$$

Then $\mathrm{H}_{1}$ and $\mathrm{H}_{2}$ are anti-hesitant fuzzy UP-subalgebras of $A$. We thus obtain the intersection of $\mathrm{H}_{1}$ and $\mathrm{H}_{2}$ as follows:

$$
\mathrm{H}_{1} \cup_{\mathrm{T}} \mathrm{H}_{2}(x)= \begin{cases}\{0.3\}, & \text { if } x=0 \\ {[0.2,0.3],} & \text { if } x=1 \\ {[0.1,0.3],} & \text { if } x=2 \\ {[0.3,0.4],} & \text { if } x=3\end{cases}
$$

Therefore, $\mathrm{H}_{1} \cup_{\mathrm{T}} \mathrm{H}_{2}$ is not an anti-hesitant fuzzy UP-subalgebra of $A$ because

$$
\mathrm{h}_{\mathrm{H}_{1} \cup \cup_{\mathrm{H}}}(1 \cdot 3)=\mathrm{h}_{\mathrm{H}_{1} \cup \mathrm{H}_{2}}(2)=[0.1,0.3] \nsubseteq(0.2,0.4]=(0.2,0.3] \cup[0.3,0.4]=\mathrm{h}_{\mathrm{H}_{1} \cup_{T} \mathrm{H}_{2}}(1) \cup \mathrm{h}_{\mathrm{H}_{1} \cup_{T} \mathrm{H}_{2}}(3) .
$$

The following example shows that the union of anti-hesitant fuzzy UP-filters of $A$ is not an antihesitant fuzzy UP-filter of $A$.

Example 4.15. Let $A=\{0,1,2,3\}$ be a set with a binary operation - defined by the following Cayley table:

$$
\begin{array}{c|cccc}
\cdot & 0 & 1 & 2 & 3 \\
\hline 0 & 0 & 1 & 2 & 3 \\
1 & 0 & 0 & 3 & 3 \\
2 & 0 & 1 & 0 & 0 \\
3 & 0 & 1 & 2 & 0
\end{array}
$$


Then $(A, \cdot, 0)$ is a UP-algebra. We define two hesitant fuzzy sets $\mathrm{H}_{1}$ and $\mathrm{H}_{2}$ on $A$ as follows:

$$
\mathrm{h}_{\mathrm{H}_{1}}(x)=\left\{\begin{array}{ll}
\emptyset, & \text { if } x=0, \\
\{0\}, & \text { if } x=1, \\
\{1\}, & \text { if } x=2, \\
\{1\}, & \text { if } x=3,
\end{array} \text {. } \quad \text { and } \mathrm{h}_{\mathrm{H}_{2}}(x)= \begin{cases}\{0.8\}, & \text { if } x=0, \\
\{0.8,1\}, & \text { if } x=1, \\
\{0,0.2,0.8\}, & \text { if } x=2, \\
\{0,0.8\}, & \text { if } x=3 .\end{cases}\right.
$$

Then $\mathrm{H}_{1}$ and $\mathrm{H}_{2}$ are anti-hesitant fuzzy UP-filters of $A$. We thus obtain the intersection of $\mathrm{H}_{1}$ and $\mathrm{H}_{2}$ as follows:

$$
\mathrm{H}_{1} \cap_{\mathrm{T}} \mathrm{H}_{2}(x)= \begin{cases}\{0.8\}, & \text { if } x=0, \\ \{0.8,1\}, & \text { if } x=1, \\ \{1\}, & \text { if } x=2, \\ \{1\}, & \text { if } x=3 .\end{cases}
$$

Therefore, $\mathrm{H}_{1} \cup_{\mathrm{T}} \mathrm{H}_{2}$ is not an anti-hesitant fuzzy UP-filter of $A$ because

$$
\mathrm{h}_{\mathrm{H}_{1} \cup_{\mathrm{T}} \mathrm{H}_{2}}(0)=\{0.8\} \nsubseteq\{1\}=\mathrm{h}_{\mathrm{H}_{1} \cup_{\mathrm{T}} \mathrm{H}_{2}}(2) \text {. }
$$

The following example shows that the union of anti-hesitant fuzzy UP-ideals of $A$ is not an antihesitant fuzzy UP-ideal of $A$.

Example 4.16. Let $A=\{0,1,2,3\}$ be a set with a binary operation - defined by the following Cayley table:

\begin{tabular}{l|llll}
$\cdot$ & 0 & 1 & 2 & 3 \\
\hline 0 & 0 & 1 & 2 & 3 \\
1 & 0 & 0 & 2 & 2 \\
2 & 0 & 1 & 0 & 1 \\
3 & 0 & 0 & 0 & 0
\end{tabular}

Then $(A, \cdot, 0)$ is a UP-algebra. We define two hesitant fuzzy sets $H_{1}$ and $H_{2}$ on $A$ as follows:

$$
\mathrm{h}_{\mathrm{H}_{1}}(x)=\left\{\begin{array}{ll}
\{0\}, & \text { if } x=0, \\
\{0\}, & \text { if } x=1, \\
\{0,0.2\}, & \text { if } x=2, \\
\{0,0.2\}, & \text { if } x=3,
\end{array} \text { and } \mathrm{h}_{\mathrm{H}_{2}}(x)= \begin{cases}\emptyset, & \text { if } x=0, \\
\{0.5\}, & \text { if } x=1, \\
\emptyset, & \text { if } x=2, \\
\{0.5\}, & \text { if } x=3 .\end{cases}\right.
$$

Then $\mathrm{H}_{1}$ and $\mathrm{H}_{2}$ are anti-hesitant fuzzy UP-ideals of $A$. We thus obtain the intersection of $\mathrm{H}_{1}$ and $\mathrm{H}_{2}$ as follows:

$$
\mathrm{H}_{1} \cup_{\mathrm{T}} \mathrm{H}_{2}(x)= \begin{cases}\{0\}, & \text { if } x=0, \\ \{0.5\}, & \text { if } x=1, \\ \{0,0.2\}, & \text { if } x=2, \\ \{0.5\}, & \text { if } x=3 .\end{cases}
$$

Therefore, $\mathrm{H}_{1} \cup_{\mathrm{T}} \mathrm{H}_{2}$ is not an anti-hesitant fuzzy UP-ideal of $A$ because

$$
\mathrm{h}_{\mathrm{H}_{1} \cup_{\mathrm{T}} \mathrm{H}_{2}}(0)=\{1\} \nsubseteq\{0.5\}=\mathrm{h}_{\mathrm{H}_{1} \cup_{\top} \mathrm{H}_{2}}(1) .
$$

Theorem 4.17. The union of anti-hesitant fuzzy strongly UP-ideals of $A$ is an anti-hesitant fuzzy strongly UPideals of A.

Proof. Assume that $\mathrm{H}$ and $\mathrm{G}$ are anti-hesitant fuzzy strongly UP-ideals of $A$. By Theorem 3.5, we have $H$ and $G$ are constant hesitant fuzzy sets of $A$. Thus $H \cup_{T} G$ is a constant hesitant fuzzy set of $A$. By Theorem 3.5, we can conclude that $\mathrm{H} \cup_{\mathrm{T}} \mathrm{G}$ is an anti-hesitant fuzzy strongly UP-ideal of $A$. 
We see that the intersection and the union can not be applied to several types of hesitant fuzzy sets on UP-algebras except hesitant fuzzy strongly UP-ideals and anti-hesitant fuzzy strongly UP-ideals. That make we will consider the others.

The concepts of the hesitant union, and the hesitant intersection of two hesitant fuzzy sets were introduced by Jun [7] in 2015. We will introduce the notions of the hesitant union and the hesitant intersection of any hesitant fuzzy sets and apply to UP-algebras.

Definition 4.18. Let $\left\{\mathrm{H}_{i} \mid i \in \mathrm{I}\right\}$ be a family of hesitant fuzzy sets on a reference set $X$. We define the hesitant intersection operation $\cap$ on $\left\{\mathrm{H}_{i} \mid i \in \mathrm{I}\right\}$ by

$$
\bigcap_{i \in \mathrm{I}} \mathrm{H}_{\mathrm{i}}=\left\{\left(x, \bigcap_{i \in \mathrm{I}} \mathrm{h}_{\mathrm{H}_{\mathrm{i}}}(x)\right) \mid x \in \mathrm{X}\right\}
$$

which is called the hesitant intersection of hesitant fuzzy sets. In particular, $\mathrm{H}_{\mathrm{i}} \cap \mathrm{H}_{\mathrm{k}}=\left\{\left(x, \mathrm{~h}_{\mathrm{H}_{\mathrm{i}}}(\mathrm{x}) \cap \mathrm{h}_{\mathrm{H}_{\mathrm{k}}}(\mathrm{x})\right) \mid\right.$ $x \in X\}$ for all $i, k \in I$.

Theorem 4.19. The hesitant intersection of hesitant fuzzy UP-subalgebras of $\mathrm{A}$ is a hesitant fuzzy UP-subalgebra of $A$.

Proof. Assume that $\left\{\mathrm{H}_{i} \mid i \in I\right\}$ is a family of hesitant fuzzy UP-subalgebras of $A$. Let $x, y \in A$. Then $h_{\mathrm{H}_{i}}(x \cdot y) \supseteq h_{H_{i}}(x) \cap h_{H_{i}}(y)$ for all $i \in I$. Thus

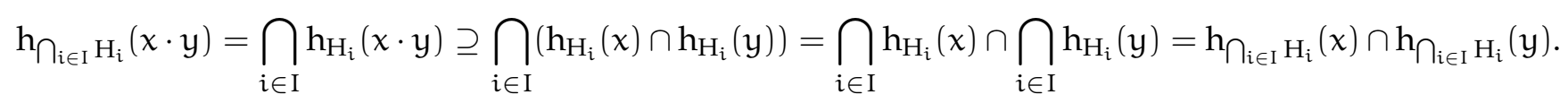

Hence, $\bigcap_{i \in \mathrm{I}} \mathrm{H}_{i}$ is a hesitant fuzzy UP-subalgebra of $A$.

Theorem 4.20. The hesitant intersection of hesitant fuzzy UP-filters of A is a hesitant fuzzy UP-filter of A.

Proof. Assume that $\left\{\mathrm{H}_{i} \mid i \in I\right\}$ is a family of hesitant fuzzy UP-filters of $A$. Let $x, y \in A$. Then $h_{H_{i}}(0) \supseteq$ $h_{H_{i}}(x)$ and $h_{H_{i}}(y) \supseteq h_{H_{i}}(x \cdot y) \cap h_{H_{i}}(x)$ for all $i \in I$. Thus $h_{\bigcap_{i \in I} H_{i}}(0)=\bigcap_{i \in I} h_{H_{i}}(0) \supseteq \bigcap_{i \in I} h_{H_{i}}(x)=h_{\bigcap_{i \in I} H_{i}}(x)$ and

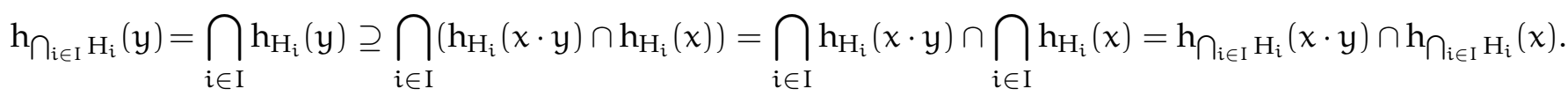

Hence, $\bigcap_{i \in I} H_{i}$ is a hesitant fuzzy UP-filter of $A$.

Theorem 4.21. The hesitant intersection of hesitant fuzzy UP-ideals of A is a hesitant fuzzy UP-ideal of A.

Proof. Assume that $\left\{\mathrm{H}_{\mathrm{i}} \mid \mathrm{i} \in \mathrm{I}\right\}$ is a family of hesitant fuzzy UP-ideals of $A$. Let $x, y \in A$. Then $\mathrm{h}_{\mathrm{H}_{\mathrm{i}}}(0) \supseteq$ $h_{H_{i}}(x)$ and $h_{H_{i}}(x \cdot z) \supseteq h_{H_{i}}(x \cdot(y \cdot z)) \cap h_{H_{i}}(y)$ for all $i \in I$. Thus $h_{\bigcap_{i} \in \mathrm{I}} H_{i}(0)=\bigcap_{i \in I} h_{H_{i}}(0) \supseteq \bigcap_{i \in I} h_{H_{i}}(x)=$ $\mathrm{h}_{\bigcap_{\mathrm{i} \in \mathrm{I}} \mathrm{H}_{\mathrm{i}}}(\mathrm{x})$ and

$$
\begin{aligned}
\mathrm{h}_{\bigcap_{i \in \mathrm{I}} \mathrm{H}_{\mathrm{i}}}(x \cdot z)=\bigcap_{i \in \mathrm{I}} \mathrm{h}_{\mathrm{H}_{\mathrm{i}}}(x \cdot z) \supseteq \bigcap_{i \in \mathrm{I}}\left(\mathrm{h}_{\mathrm{H}_{\mathrm{i}}}(x \cdot(y \cdot z)) \cap \mathrm{h}_{\mathrm{H}_{\mathrm{i}}}(\mathrm{y})\right) & =\bigcap_{\mathrm{i} \in \mathrm{I}} \mathrm{h}_{\mathrm{H}_{\mathrm{i}}}(\mathrm{x} \cdot(\mathrm{y} \cdot z)) \cap \bigcap_{\mathrm{i} \in \mathrm{I}} \mathrm{h}_{\mathrm{H}_{\mathrm{i}}}(\mathrm{y}) \\
& =\mathrm{h}_{\bigcap_{\mathrm{i} \in \mathrm{I}} \mathrm{H}_{\mathrm{i}}}(\mathrm{x} \cdot(\mathrm{y} \cdot z)) \cap \mathrm{h}_{\bigcap_{\mathrm{i} \in \mathrm{I}} \mathrm{H}_{\mathrm{i}}}(\mathrm{y}) .
\end{aligned}
$$

Hence, $\bigcap_{i \in I} H_{i}$ is a hesitant fuzzy UP-ideal of $A$. 
Theorem 4.22. The hesitant intersection of hesitant fuzzy strongly UP-ideals of $\mathrm{A}$ is a hesitant fuzzy strongly UP-ideal of A.

Proof. Assume that $\left\{\mathrm{H}_{i} \mid i \in \mathrm{I}\right\}$ is a family of hesitant fuzzy strongly UP-ideals of $A$. Then $\mathrm{H}_{i}$ is a hesitant fuzzy strongly UP-ideal of $A$ for all $i \in I$. By Theorem 3.3, we have $H_{i}$ is a constant hesitant fuzzy set on A for all $i \in I$. Thus $\bigcap_{i \in I} H_{i}$ is a constant hesitant fuzzy set on $A$. By Theorem 3.3, we know that $\bigcap_{i \in I} H_{i}$ is a hesitant fuzzy strongly UP-ideal of $A$.

The following example shows that the hesitant intersection of anti-hesitant fuzzy UP-subalgebras of $A$ is not an anti-hesitant fuzzy UP-subalgebra of $A$.

Example 4.23. Let $A=\{0,1,2,3\}$ be a set with a binary operation - defined by the following Cayley table:

\begin{tabular}{l|llll}
$\cdot$ & 0 & 1 & 2 & 3 \\
\hline 0 & 0 & 1 & 2 & 3 \\
1 & 0 & 0 & 2 & 2 \\
2 & 0 & 1 & 0 & 1 \\
3 & 0 & 0 & 0 & 0
\end{tabular}

Then $(A, \cdot, 0)$ is a UP-algebra. We define two hesitant fuzzy sets $\mathrm{H}_{1}$ and $\mathrm{H}_{2}$ on $A$ as follows:

$$
h_{\mathrm{H}_{1}}(x)=\left\{\begin{array}{ll}
\{0.3\}, & \text { if } x=0, \\
{[0.3,0.4),} & \text { if } x=1, \\
{[0.3,0.5],} & \text { if } x=2, \\
{[0,0.5],} & \text { if } x=3,
\end{array} \text {, } \quad \text { and } h_{\mathrm{H}_{2}}(x)= \begin{cases}\{0.3\}, & \text { if } x=0, \\
\{0.3,0.4\}, & \text { if } x=1, \\
\{0.3,0.4\}, & \text { if } x=2, \\
\{0.3\}, & \text { if } x=3 .\end{cases}\right.
$$

Then $\mathrm{H}_{1}$ and $\mathrm{H}_{2}$ are anti-hesitant fuzzy UP-subalgebras of $A$. We thus obtain the hesitant intersection of $\mathrm{H}_{1}$ and $\mathrm{H}_{2}$ as follows:

$$
\mathrm{h}_{\mathrm{H}_{1} \cap \mathrm{H}_{2}}(x)= \begin{cases}\{0.3\}, & \text { if } x=0 \\ \{0.3\}, & \text { if } x=1, \\ \{0.3,0.4\}, & \text { if } x=2, \\ \{0.3\}, & \text { if } x=3\end{cases}
$$

Therefore, $\mathrm{H}_{1} \cap \mathrm{H}_{2}$ is not an anti-hesitant fuzzy UP-subalgebra of $A$ because

$$
\mathrm{h}_{\mathrm{H}_{1} \cap \mathrm{H}_{2}}(1 \cdot 3)=\mathrm{h}_{\mathrm{H}_{1} \cap \mathrm{H}_{2}}(2)=\{0.3,0.4\} \nsubseteq\{0.3\}=\{0.3\} \cup\{0.3\}=\mathrm{h}_{\mathrm{H}_{1} \cap \mathrm{H}_{2}}(1) \cup \mathrm{h}_{\mathrm{H}_{1} \cap \mathrm{H}_{2}}(3) .
$$

The following example shows that the hesitant intersection of anti-hesitant fuzzy UP-filters of $A$ is not an anti-hesitant fuzzy UP-filter of A.

Example 4.24. Let $A=\{0,1,2,3\}$ be a set with a binary operation - defined by the following Cayley table:

$$
\begin{array}{l|llll} 
& 0 & 1 & 2 & 3 \\
\hline 0 & 0 & 1 & 2 & 3 \\
1 & 0 & 0 & 3 & 3 \\
2 & 0 & 1 & 0 & 0 \\
3 & 0 & 1 & 2 & 0
\end{array}
$$

Then $(A, \cdot, 0)$ is a UP-algebra. We define two hesitant fuzzy sets $H_{1}$ and $H_{2}$ on $A$ as follows:

$$
h_{\mathrm{H}_{1}}(x)=\left\{\begin{array}{ll}
\{0.6\}, & \text { if } x=0, \\
\{0.6,0.7\}, & \text { if } x=1, \\
\{0.5,0.6\}, & \text { if } x=2, \\
\{0.5,0.6\}, & \text { if } x=3,
\end{array} \text {, nd } h_{\mathrm{H}_{2}}(x)= \begin{cases}\{0.6\}, & \text { if } x=0, \\
\{0.5,0.6,0.7\}, & \text { if } x=1, \\
\{0.4,0.5,0.6\}, & \text { if } x=2, \\
\{0.4,0.6\}, & \text { if } x=3\end{cases}\right.
$$


Then $\mathrm{H}_{1}$ and $\mathrm{H}_{2}$ are anti-hesitant fuzzy UP-filters of $A$. We thus obtain the hesitant intersection of $\mathrm{H}_{1}$ and $\mathrm{H}_{2}$ as follows:

$$
\mathrm{h}_{\mathrm{H}_{1} \cap \mathrm{H}_{2}}(\mathrm{x})= \begin{cases}\{0.6\}, & \text { if } \mathrm{x}=0 \\ \{0.6,0.7\}, & \text { if } \mathrm{x}=1 \\ \{0.5,0.6\}, & \text { if } \mathrm{x}=2 \\ \{0.6\}, & \text { if } x=3\end{cases}
$$

Therefore, $\mathrm{H}_{1} \cap \mathrm{H}_{2}$ is not an anti-hesitant fuzzy UP-filter of $A$ because

$$
\mathrm{h}_{\mathrm{H}_{1} \cap \mathrm{H}_{2}}(2)=\{0.5,0.6\} \nsubseteq\{0.6,0.7\}=\{0.6\} \cup\{0.6,0.7\}=\mathrm{h}_{\mathrm{H}_{1} \cap \mathrm{H}_{2}}(3) \cup \mathrm{h}_{\mathrm{H}_{1} \cap \mathrm{H}_{2}}(1)=\mathrm{h}_{\mathrm{H}_{1} \cap \mathrm{H}_{2}}(1 \cdot 2) \cup \mathrm{h}_{\mathrm{H}_{1} \cap \mathrm{H}_{2}}(1) .
$$

The following example shows that the hesitant intersection of anti-hesitant fuzzy UP-ideals of $A$ is not an anti-hesitant fuzzy UP-ideal of $A$.

Example 4.25. Let $A=\{0,1,2,3\}$ be a set with a binary operation - defined by the following Cayley table:

\begin{tabular}{l|llll}
$\cdot$ & 0 & 1 & 2 & 3 \\
\hline 0 & 0 & 1 & 2 & 3 \\
1 & 0 & 0 & 2 & 2 \\
2 & 0 & 1 & 0 & 1 \\
3 & 0 & 0 & 0 & 0
\end{tabular}

Then $(A, \cdot, 0)$ is a UP-algebra. We define two hesitant fuzzy sets $\mathrm{H}_{1}$ and $\mathrm{H}_{2}$ on $A$ as follows:

$$
h_{\mathrm{H}_{1}}(x)=\left\{\begin{array}{ll}
\{0.9\}, & \text { if } x=0, \\
\{0.9\}, & \text { if } x=1, \\
\{0.9,1\}, & \text { if } x=2, \\
\{0.9,1\}, & \text { if } x=3,
\end{array} \text {, } \quad \text { and } h_{\mathrm{H}_{2}}(x)= \begin{cases}\{0.9\}, & \text { if } x=0, \\
\{0.9,1\}, & \text { if } x=1, \\
\{0.9\}, & \text { if } x=2, \\
\{0.9,1\}, & \text { if } x=3 .\end{cases}\right.
$$

Then $\mathrm{H}_{1}$ and $\mathrm{H}_{2}$ are anti-hesitant fuzzy UP-ideals of $A$. We thus obtain the hesitant intersection of $\mathrm{H}_{1}$ and $\mathrm{H}_{2}$ as follows:

$$
\mathrm{h}_{\mathrm{H}_{1} \cap \mathrm{H}_{2}}(x)= \begin{cases}\{0.9\}, & \text { if } x=0 \\ \{0.9\}, & \text { if } x=1 \\ \{0.9\}, & \text { if } x=2 \\ \{0.9,1\}, & \text { if } x=3\end{cases}
$$

Therefore, $\mathrm{H}_{1} \cap \mathrm{H}_{2}$ is not an anti-hesitant fuzzy UP-ideal of $A$ because

$$
\begin{aligned}
\mathrm{h}_{\mathrm{H}_{1} \cap \mathrm{H}_{2}}(0 \cdot 3)=\mathrm{h}_{\mathrm{H}_{1} \cap \mathrm{H}_{2}}(3)=\{0.9,1\} \nsubseteq\{0.9\} & =\{0.9\} \cup\{0.9\} \\
& =\mathrm{h}_{\mathrm{H}_{1} \cap \mathrm{H}_{2}}(1) \cup \mathrm{h}_{\mathrm{H}_{1} \cap \mathrm{H}_{2}}(2)=\mathrm{h}_{\mathrm{H}_{1} \cap \mathrm{H}_{2}}(0 \cdot(2 \cdot 3)) \cup \mathrm{h}_{\mathrm{H}_{1} \cap \mathrm{H}_{2}}(2) .
\end{aligned}
$$

Theorem 4.26. The hesitant intersection of anti-hesitant fuzzy strongly UP-ideals of $\mathrm{A}$ is an anti-hesitant fuzzy strongly UP-ideal of A.

Proof. Assume that $\left\{\mathrm{H}_{i} \mid i \in \mathrm{I}\right\}$ is a family of anti-hesitant fuzzy strongly UP-ideals of $A$. Then $\mathrm{H}_{i}$ is an anti-hesitant fuzzy strongly UP-ideal of $A$ for all $i \in \mathrm{I}$. By Theorem 3.5, we have $\mathrm{H}_{i}$ is a constant hesitant fuzzy set on $A$ for all $i \in I$. Thus $\bigcap_{i \in I} H_{i}$ is a constant hesitant fuzzy set on $A$. By Theorem 3.5, we know that $\bigcap_{i \in \mathrm{I}} \mathrm{H}_{i}$ is an anti-hesitant fuzzy strongly UP-ideal of $A$.

Definition 4.27. Let $\left\{\mathrm{H}_{i} \mid i \in \mathrm{I}\right\}$ be a family of hesitant fuzzy sets on a reference set $X$. We define the hesitant union operation $\cup$ on $\left\{\mathrm{H}_{\mathrm{i}} \mid \mathrm{i} \in \mathrm{I}\right\}$ by

$$
\bigcup_{i \in \mathrm{I}} \mathrm{H}_{\mathrm{i}}=\left\{\left(x, \bigcup_{i \in \mathrm{I}} \mathrm{h}_{\mathrm{H}_{\mathrm{i}}}(x)\right) \mid x \in X\right\},
$$

which is called the hesitant union of hesitant fuzzy sets. In particular, $\mathrm{H}_{i} \cup \mathrm{H}_{\mathrm{k}}=\left\{\left(x, \mathrm{~h}_{\mathrm{H}_{\mathrm{i}}}(x) \cup \mathrm{h}_{\mathrm{H}_{\mathrm{k}}}(\mathrm{x})\right) \mid \mathrm{x} \in\right.$ $X\}$ for all $i, k \in I$. 
The following example shows that the hesitant union of hesitant fuzzy UP-subalgebras of $A$ is not a hesitant fuzzy UP-subalgebra of $A$.

Example 4.28. Let $A=\{0,1,2,3\}$ be a set with a binary operation - defined by the following Cayley table:

\begin{tabular}{l|llll}
$\cdot$ & 0 & 1 & 2 & 3 \\
\hline 0 & 0 & 1 & 2 & 3 \\
1 & 0 & 0 & 2 & 2 \\
2 & 0 & 1 & 0 & 1 \\
3 & 0 & 0 & 0 & 0
\end{tabular}

Then $(A, \cdot, 0)$ is a UP-algebra. We define two hesitant fuzzy sets $H_{1}$ and $H_{2}$ on $A$ as follows:

$$
\mathrm{h}_{\mathrm{H}_{1}}(x)=\left\{\begin{array}{ll}
{[0.6,0.9],} & \text { if } x=0, \\
\{0.8\}, & \text { if } x=1, \\
\{0.7\}, & \text { if } x=2, \\
\{0.6\}, & \text { if } x=3,
\end{array} \text {, } \quad \text { and } \mathrm{h}_{\mathrm{H}_{2}}(x)= \begin{cases}{[0.6,0.8],} & \text { if } x=0, \\
\{0.7\}, & \text { if } x=1, \\
\{0.7\}, & \text { if } x=2, \\
{[0.6,0.8],} & \text { if } x=3 .\end{cases}\right.
$$

Then $\mathrm{H}_{1}$ and $\mathrm{H}_{2}$ are hesitant fuzzy UP-subalgebras of $A$. We thus obtain the hesitant union of $\mathrm{H}_{1}$ and $\mathrm{H}_{2}$ as follows:

$$
h_{\mathrm{H}_{1} \cup \mathrm{H}_{2}}(x)= \begin{cases}{[0.6,0.9],} & \text { if } x=0 \\ \{0.7,0.8\}, & \text { if } x=1 \\ \{0.7\}, & \text { if } x=2 \\ {[0.6,0.8],} & \text { if } x=3\end{cases}
$$

Therefore, $\mathrm{H}_{1} \cup \mathrm{H}_{2}$ is not a hesitant fuzzy UP-subalgebra of $A$ because

$$
\mathrm{h}_{\mathrm{H}_{1} \cup \mathrm{H}_{2}}(1 \cdot 3)=\mathrm{h}_{\mathrm{H}_{1} \cap \mathrm{H}_{2}}(2)=\{0.7\} \nsupseteq\{0.7,0.8\}=\{0.7,0.8\} \cap[0.6,0.8]=\mathrm{h}_{\mathrm{H}_{1} \cup \mathrm{H}_{2}}(1) \cap \mathrm{h}_{\mathrm{H}_{1} \cup \mathrm{H}_{2}}(3) .
$$

The following example shows that the hesitant union of hesitant fuzzy UP-filters of $A$ is not a hesitant fuzzy UP-filter of $A$.

Example 4.29. Let $A=\{0,1,2,3\}$ be a set with a binary operation - defined by the following Cayley table:

$$
\begin{array}{c|cccc}
\cdot & 0 & 1 & 2 & 3 \\
\hline 0 & 0 & 1 & 2 & 3 \\
1 & 0 & 0 & 3 & 3 \\
2 & 0 & 1 & 0 & 0 \\
3 & 0 & 1 & 2 & 0
\end{array}
$$

Then $(A, \cdot, 0)$ is a UP-algebra. We define two hesitant fuzzy sets $\mathrm{H}_{1}$ and $\mathrm{H}_{2}$ on $A$ as follows:

$$
h_{\mathrm{H}_{1}}(x)=\left\{\begin{array}{ll}
\{0\} \cup[0.8,1], & \text { if } x=0, \\
\{0\} \cup[0.8,1], & \text { if } x=1, \\
\{0.8\}, & \text { if } x=2, \\
\{0.8\}, & \text { if } x=3,
\end{array} \text {, nd } h_{\mathrm{H}_{2}}(x)= \begin{cases}{[0,0.8],} & \text { if } x=0, \\
\{0.8\}, & \text { if } x=1, \\
\emptyset, & \text { if } x=2, \\
\{0\}, & \text { if } x=3 .\end{cases}\right.
$$

Then $\mathrm{H}_{1}$ and $\mathrm{H}_{2}$ are hesitant fuzzy UP-filters of $A$. We thus obtain the hesitant union of $\mathrm{H}_{1}$ and $\mathrm{H}_{2}$ as follows:

$$
\mathrm{h}_{\mathrm{H}_{1} \cup \mathrm{H}_{2}}(x)= \begin{cases}{[0,1],} & \text { if } x=0, \\ \{0\} \cup[0.8,1], & \text { if } x=1, \\ \{0.8\}, & \text { if } x=2, \\ \{0,0.8\}, & \text { if } x=3 .\end{cases}
$$

Therefore, $\mathrm{H}_{1} \cup \mathrm{H}_{2}$ is not a hesitant fuzzy UP-filter of $A$ because

$$
\mathrm{h}_{\mathrm{H}_{1} \cup \mathrm{H}_{2}}(2)=\{0.8\} \nsupseteq\{0,0.8\}=\{0,0.8\} \cap(\{0\} \cup[0.8,1])=\mathrm{h}_{\mathrm{H}_{1} \cup \mathrm{H}_{2}}(3) \cap \mathrm{h}_{\mathrm{H}_{1} \cup \mathrm{H}_{2}}(1)=\mathrm{h}_{\mathrm{H}_{1} \cup \mathrm{H}_{2}}(1 \cdot 2) \cap \mathrm{h}_{\mathrm{H}_{1} \cup \mathrm{H}_{2}}(1) .
$$


The following example shows that the hesitant union of hesitant fuzzy UP-ideals of $A$ is not a hesitant fuzzy UP-ideal of $A$.

Example 4.30. Let $A=\{0,1,2,3\}$ be a set with a binary operation - defined by the following Cayley table:

\begin{tabular}{l|llll}
$\cdot$ & 0 & 1 & 2 & 3 \\
\hline 0 & 0 & 1 & 2 & 3 \\
1 & 0 & 0 & 2 & 2 \\
2 & 0 & 1 & 0 & 1 \\
3 & 0 & 0 & 0 & 0
\end{tabular}

Then $(A, \cdot, 0)$ is a UP-algebra. We define two hesitant fuzzy sets $\mathrm{H}_{1}$ and $\mathrm{H}_{2}$ on $A$ as follows:

$$
\mathrm{h}_{\mathrm{H}_{1}}(x)=\left\{\begin{array}{ll}
\{0.9,1\}, & \text { if } x=0, \\
\{0.9,1\}, & \text { if } x=1, \\
\{1\}, & \text { if } x=2, \\
\{1\}, & \text { if } x=3,
\end{array} \text {. nd } \mathrm{h}_{\mathrm{H}_{2}}(x)= \begin{cases}\{0.9,1\}, & \text { if } x=0, \\
\{1\}, & \text { if } x=1, \\
\{0.9,1\}, & \text { if } x=2, \\
\{1\}, & \text { if } x=3\end{cases}\right.
$$

Then $\mathrm{H}_{1}$ and $\mathrm{H}_{2}$ are hesitant fuzzy UP-ideals of $A$. We thus obtain the hesitant union of $\mathrm{H}_{1}$ and $\mathrm{H}_{2}$ as follows:

$$
\mathrm{h}_{\mathrm{H}_{1} \cup \mathrm{H}_{2}}(x)= \begin{cases}\{0.9,1\}, & \text { if } x=0, \\ \{0.9,1\}, & \text { if } x=1, \\ \{0.9,1\}, & \text { if } x=2, \\ \{1\}, & \text { if } x=3 .\end{cases}
$$

Therefore, $\mathrm{H}_{1} \cup \mathrm{H}_{2}$ is not a hesitant fuzzy UP-ideal of $A$ because

$$
\begin{aligned}
\mathrm{h}_{\mathrm{H}_{1} \cup \mathrm{H}_{2}}(0 \cdot 3)=\mathrm{h}_{\mathrm{H}_{1} \cup \mathrm{H}_{2}}(3)=\{1\} \nsupseteq\{0.9,1\} & =\{0.9,1\} \cap\{0.9,1\} \\
& =\mathrm{h}_{\mathrm{H}_{1} \cup \mathrm{H}_{2}}(1) \cap \mathrm{h}_{\mathrm{H}_{1} \cup \mathrm{H}_{2}}(2)=\mathrm{h}_{\mathrm{H}_{1} \cup \mathrm{H}_{2}}(0 \cdot(2 \cdot 3)) \cap \mathrm{h}_{\mathrm{H}_{1} \cup \mathrm{H}_{2}}(2) .
\end{aligned}
$$

Theorem 4.31. The hesitant union of hesitant fuzzy strongly UP-ideals of $A$ is a hesitant fuzzy strongly UP-ideal of $A$.

Proof. Assume that $\left\{\mathrm{H}_{i} \mid i \in \mathrm{I}\right\}$ is a family of hesitant fuzzy strongly UP-ideals of $A$. Then $\mathrm{H}_{i}$ is a hesitant fuzzy strongly UP-ideal of $A$ for all $i \in I$. By Theorem 3.3, we have $\mathrm{H}_{i}$ is a constant hesitant fuzzy set on A for all $i \in I$. Thus $\bigcup_{i \in I} H_{i}$ is a constant hesitant fuzzy set on $A$. By Theorem 3.3, we know that $\bigcup_{i \in I} H_{i}$ is a hesitant fuzzy strongly UP-ideal of A.

Theorem 4.32. The hesitant union of anti-hesitant fuzzy UP-subalgebras of $\mathrm{A}$ is an anti-hesitant fuzzy UPsubalgebra of $\mathrm{A}$.

Proof. Assume that $\left\{\mathrm{H}_{i} \mid i \in \mathrm{I}\right\}$ is a family of anti-hesitant fuzzy UP-subalgebras of $A$. Let $x, y \in A$. Then $h_{\mathrm{H}_{i}}(x \cdot y) \subseteq h_{H_{i}}(x) \cup h_{H_{i}}(y)$ for all $i \in I$. Thus

$$
h_{\bigcup_{i} \in \mathrm{I}} H_{i}(x \cdot y)=\bigcup_{i \in I} h_{H_{i}}(x \cdot y) \subseteq \bigcup_{i \in I}\left(h_{H_{i}}(x) \cup h_{H_{i}}(y)\right)=\bigcup_{i \in I} h_{H_{i}}(x) \cup \bigcup_{i \in I} h_{H_{i}}(y)=h_{\cup_{i \in I} H_{i}}(x) \cup h_{\bigcup_{i \in I} H_{i}}(y) .
$$

Hence, $\bigcup_{i \in \mathrm{I}} \mathrm{H}_{i}$ is an anti-hesitant fuzzy UP-subalgebra of $A$.

Theorem 4.33. The hesitant union of anti-hesitant fuzzy UP-filters of $\mathrm{A}$ is an anti-hesitant fuzzy UP-filter of A. 
Proof. Assume that $\left\{\mathrm{H}_{i} \mid i \in \mathrm{I}\right\}$ is a family of anti-hesitant fuzzy UP-filters of $A$. Let $x, y \in A$. Then $h_{H_{i}}(0) \subseteq h_{H_{i}}(x)$ and $h_{H_{i}}(y) \subseteq h_{H_{i}}(x \cdot y) \cup h_{H_{i}}(x)$ for all $i \in I$. Thus $h_{\cup_{i \in I} H_{i}}(0)=\bigcup_{i \in I} h_{H_{i}}(0) \subseteq \bigcup_{i \in I} h_{H_{i}}(x)=$ $h_{\cup_{i \in I} H_{i}}(x)$ and

$h_{\bigcup_{i \in I} H_{i}}(y)=\bigcup_{i \in I} h_{H_{i}}(y) \subseteq \bigcup_{i \in I}\left(h_{H_{i}}(x \cdot y) \cup h_{H_{i}}(x)\right)=\bigcup_{i \in I} h_{H_{i}}(x \cdot y) \cup \bigcup_{i \in I} h_{H_{i}}(x)=h_{\bigcup_{i \in I} H_{i}}(x \cdot y) \cup h_{\bigcup_{i \in I} H_{i}}(x)$.

Hence, $\bigcup_{i \in I} H_{i}$ is an anti-hesitant fuzzy UP-filter of $A$.

Theorem 4.34. The hesitant union of anti-hesitant fuzzy UP-ideals of $\mathrm{A}$ is an anti-hesitant fuzzy UP-ideal of A.

Proof. Assume that $\left\{\mathrm{H}_{i} \mid i \in \mathrm{I}\right\}$ is a family of anti-hesitant fuzzy UP-ideals of $A$. Let $x, y \in A$. Then $\mathrm{h}_{\mathrm{H}_{\mathrm{i}}}(0) \subseteq \mathrm{h}_{\mathrm{H}_{\mathrm{i}}}(\mathrm{x})$ and $\mathrm{h}_{\mathrm{H}_{\mathrm{i}}}(\mathrm{x} \cdot z) \subseteq \mathrm{h}_{\mathrm{H}_{\mathrm{i}}}(\mathrm{x} \cdot(\mathrm{y} \cdot z)) \cup \mathrm{h}_{\mathrm{H}_{\mathrm{i}}}(\mathrm{y})$ for all $\mathrm{i} \in \mathrm{I}$. Thus $\mathrm{h}_{\cup_{\mathrm{i} \in \mathrm{I}} \mathrm{H}_{\mathrm{i}}}(0)=\bigcup_{i \in \mathrm{I}} \mathrm{h}_{\mathrm{H}_{\mathrm{i}}}(0) \subseteq$ $\bigcup_{i \in \mathrm{I}} \mathrm{h}_{\mathrm{H}_{\mathrm{i}}}(x)=\mathrm{h}_{\cup_{i \in \mathrm{I}} \mathrm{H}_{\mathrm{i}}}(x)$ and

$$
\begin{aligned}
h_{\cup_{i \in I} H_{i}}(x \cdot z)=\bigcup_{i \in I} h_{H_{i}}(x \cdot z) & \subseteq \bigcup_{i \in I}\left(h_{H_{i}}(x \cdot(y \cdot z)) \cup h_{H_{i}}(y)\right) \\
& =\bigcup_{i \in I} h_{H_{i}}(x \cdot(y \cdot z)) \cup \bigcup_{i \in I} h_{H_{i}}(y)=h_{\bigcup_{i \in I} H_{i}}(x \cdot(y \cdot z)) \cup h_{\bigcup_{i \in I} H_{i}}(y) .
\end{aligned}
$$

Hence, $\bigcup_{i \in I} H_{i}$ is an anti-hesitant fuzzy UP-ideal of $A$.

Theorem 4.35. The hesitant union of anti-hesitant fuzzy strongly UP-ideals of $\mathrm{A}$ is an anti-hesitant fuzzy strongly UP-ideal of $A$.

Proof. Assume that $\left\{\mathrm{H}_{i} \mid i \in \mathrm{I}\right\}$ is a family of anti-hesitant fuzzy strongly UP-ideals of $A$. Then $\mathrm{H}_{i}$ is an anti-hesitant fuzzy strongly UP-ideal of $A$ for all $i \in \mathrm{I}$. By Theorem 3.5, we have $\mathrm{H}_{i}$ is a constant hesitant fuzzy set on $A$ for all $i \in I$. Thus $\bigcup_{i \in I} H_{i}$ is a constant hesitant fuzzy set on $A$. By Theorem 3.5, we know that $\bigcup_{i \in I} H_{i}$ is an anti-hesitant fuzzy strongly UP-ideal of $A$.

\section{Operations on hesitant fuzzy soft sets}

The concepts of the "AND" operation and the "OR" operation on two hesitant fuzzy soft sets and the union and the intersection of two hesitant fuzzy soft sets were introduced by Wang et al. [33] in 2014.

Definition 5.1 ([33]). Let $\left(\widetilde{H}_{1}, Y_{1}\right)$ and $\left(\widetilde{H}_{2}, Y_{2}\right)$ be two hesitant fuzzy soft sets over a reference set $X$. The AND operation on $\left(\widetilde{H}_{1}, Y_{1}\right)$ and $\left(\widetilde{H}_{2}, Y_{2}\right)$ is defined to be the hesitant fuzzy soft sets $(\widetilde{H}, Y)$ satisfying the following properties:

(1) $Y=Y_{1} \times Y_{2}$, and

(2) $\tilde{\mathrm{H}}\left[\mathrm{p}_{1}, \mathrm{p}_{2}\right]=\widetilde{\mathrm{H}}_{1}\left[\mathrm{p}_{1}\right] \cap \widetilde{\mathrm{H}}_{2}\left[\mathrm{p}_{2}\right]$ for any $\left(\mathrm{p}_{1}, \mathrm{p}_{2}\right) \in \mathrm{Y}$.

We write $\left(\widetilde{\mathrm{H}}_{1}, \mathrm{Y}_{1}\right) \widetilde{\wedge}\left(\widetilde{\mathrm{H}}_{2}, \mathrm{Y}_{2}\right)=(\widetilde{\mathrm{H}}, \mathrm{Y})$.

Definition 5.2 ([33]). Let $\left(\tilde{\mathrm{H}}_{1}, \mathrm{Y}_{1}\right)$ and $\left(\tilde{\mathrm{H}}_{2}, \mathrm{Y}_{2}\right)$ be two hesitant fuzzy soft sets over a reference set $\mathrm{X}$. The OR operation on $\left(\widetilde{H}_{1}, Y_{1}\right)$ and $\left(\widetilde{H}_{2}, Y_{2}\right)$ is defined to be the hesitant fuzzy soft sets $(\widetilde{H}, Y)$ satisfying the following properties: 
(1) $Y=Y_{1} \times Y_{2}$, and

(2) $\widetilde{\mathrm{H}}\left[\mathrm{p}_{1}, \mathrm{p}_{2}\right]=\widetilde{\mathrm{H}}_{1}\left[\mathrm{p}_{1}\right] \cup \widetilde{\mathrm{H}}_{2}\left[\mathrm{p}_{2}\right]$ for any $\left(\mathrm{p}_{1}, \mathrm{p}_{2}\right) \in \mathrm{Y}$.

We write $\left(\widetilde{\mathrm{H}}_{1}, \mathrm{Y}_{1}\right) \widetilde{\mathrm{V}}\left(\widetilde{\mathrm{H}}_{2}, \mathrm{Y}_{2}\right)=(\widetilde{\mathrm{H}}, \mathrm{Y})$.

Definition 5.3 ([33]). Let $\left(\widetilde{\mathrm{H}}_{1}, \mathrm{Y}_{1}\right)$ and $\left(\widetilde{\mathrm{H}}_{2}, \mathrm{Y}_{2}\right)$ be two hesitant fuzzy soft sets over a reference set $X$. The union of $\left(\widetilde{H}_{1}, Y_{1}\right)$ and $\left(\widetilde{H}_{2}, Y_{2}\right)$ is defined to be the hesitant fuzzy soft sets $(\widetilde{H}, Y)$ satisfying the following properties:

(1) $Y=Y_{1} \cup Y_{2}$, and

(2) for any $p \in Y$,

$$
\widetilde{\mathrm{H}}[p]= \begin{cases}\widetilde{\mathrm{H}}_{1}[p], & \text { if } p \in Y_{1} \backslash Y_{2}, \\ \widetilde{\mathrm{H}}_{2}[p], & \text { if } p \in Y_{2} \backslash Y_{1}, \\ \widetilde{\mathrm{H}}_{1}[p] \cup \widetilde{\mathrm{H}}_{2}[p], & \text { if } p \in Y_{1} \cap Y_{2} .\end{cases}
$$

We write $\left(\widetilde{\mathrm{H}}_{1}, \mathrm{Y}_{1}\right) \widetilde{\cup}\left(\widetilde{\mathrm{H}}_{2}, \mathrm{Y}_{2}\right)=(\widetilde{\mathrm{H}}, \mathrm{Y})$.

Definition 5.4 ([33]). Let $\left(\widetilde{H}_{1}, Y_{1}\right)$ and $\left(\widetilde{H}_{2}, Y_{2}\right)$ be two hesitant fuzzy soft sets over a reference set $X$. The intersection of $\left(\widetilde{H}_{1}, Y_{1}\right)$ and $\left(\widetilde{H}_{2}, Y_{2}\right)$ is defined to be the hesitant fuzzy soft sets $(\widetilde{H}, Y)$ satisfying the following properties:

(1) $Y=Y_{1} \cap Y_{2} \neq \emptyset$, and

(2) $\widetilde{\mathrm{H}}[\mathrm{p}]=\widetilde{\mathrm{H}}_{1}[\mathrm{p}] \cap \widetilde{\mathrm{H}}_{2}[\mathrm{p}]$ for all $\mathrm{p} \in \mathrm{Y}$.

We write $\left(\widetilde{\mathrm{H}}_{1}, \mathrm{Y}_{1}\right) \widetilde{\cap}_{\mathrm{r}}\left(\widetilde{\mathrm{H}}_{2}, \mathrm{Y}_{2}\right)=(\widetilde{\mathrm{H}}, \mathrm{Y})$.

We will introduce the notions of the union, the intersection, the restricted union, the extended intersection, the AND, and the OR of any hesitant fuzzy soft sets and apply to UP-algebras.

Definition 5.5. Let $\left\{\left(\widetilde{H}_{i}, Y_{i}\right) \mid i \in I\right\}$ be a family of hesitant fuzzy soft sets over a reference set $X$. The restricted union of $\left(\widetilde{H}_{i}, Y_{i}\right)$ is defined to be the hesitant fuzzy soft sets $(\widetilde{H}, Y)$ satisfying the following properties:

(1) $Y=\bigcap_{i \in I} Y_{i} \neq \emptyset$, and

(2) $\widetilde{H}[p]=\bigcup_{i \in I} \widetilde{H}_{i}[p]$ for all $p \in Y$.

We write $\widetilde{\bigcup_{i \in I}} r\left(\widetilde{H}_{i}, Y_{i}\right)=(\widetilde{H}, Y)$.

Theorem 5.6. The restricted union of anti-hesitant fuzzy soft UP-subalgebras of $A$ is an anti-hesitant fuzzy soft UP-subalgebra of A.

Proof. Let $\left(\widetilde{H}_{i}, Y_{i}\right)$ be an anti-hesitant fuzzy soft UP-subalgebra of $A$ for all $i \in I$. Then $\widetilde{H}_{i}\left[p_{i}\right]$ is an antihesitant fuzzy UP-subalgebras of $A$ for all $p_{i} \in Y_{i}$ and $i \in I$. Assume that $(\widetilde{H}, Y)$ is the restricted union of $\left(\widetilde{H}_{i}, Y_{i}\right)$ for all $i \in I$ and let $p \in Y$. Then $p \in Y=\bigcap_{i \in I} Y_{i} \neq \emptyset$ and $\widetilde{H}[p]=\bigcup_{i \in I} \widetilde{H}_{i}[p]$. Thus $p \in Y_{i}$ for all $i \in I$ and so $\widetilde{H}_{i}[p]$ is a hesitant fuzzy UP-subalgebras of $A$ for all $i \in I$. By Theorem 4.32, we have $\widetilde{\mathrm{H}}[\mathrm{p}]=\bigcup_{i \in \mathrm{I}} \widetilde{\mathrm{H}}_{i}[\mathrm{p}]$ is a hesitant fuzzy UP-subalgebra of $A$. Therefore, $(\widetilde{\mathrm{H}}, \mathrm{Y})$ is a $p$-hesitant fuzzy soft UP-subalgebra of $A$. Since $p$ is arbitrary, we know that $(\widetilde{H}, Y)$ is a hesitant fuzzy soft UP-subalgebra of A. 
In the same way as Theorem 5.6, we can use Theorems $4.33,4.34,4.35,4.31$, respectively, to prove the following theorems.

Theorem 5.7. The restricted union of anti-hesitant fuzzy soft UP-filters of $A$ is an anti-hesitant fuzzy soft UP-filter of $A$.

Theorem 5.8. The restricted union of anti-hesitant fuzzy soft UP-ideals of $\mathrm{A}$ is an anti-hesitant fuzzy soft UP-ideal of $A$.

Theorem 5.9. The restricted union of anti-hesitant fuzzy soft strongly UP-ideals of $A$ is an anti-hesitant fuzzy soft strongly UP-ideal of A.

Theorem 5.10. The restricted union of hesitant fuzzy soft strongly UP-ideals of $\mathrm{A}$ is a hesitant fuzzy soft strongly UP-ideal of $A$.

By Example 4.28 (resp., Example 4.29, Example 4.30), we can imply that the restricted union of hesitant fuzzy soft UP-subalgebras (resp., hesitant fuzzy soft UP-filters, hesitant fuzzy soft UP-ideals) of $A$ is not a hesitant fuzzy soft UP-subalgebra (resp., hesitant fuzzy soft UP-filter, hesitant fuzzy soft UP-ideal) of $A$ in general.

Definition 5.11. Let $\left\{\left(\widetilde{H}_{i}, Y_{i}\right) \mid i \in I\right\}$ be a family of hesitant fuzzy soft sets over a reference set $X$. The union of $\left(\widetilde{H}_{i}, Y_{i}\right)$ is defined to be the hesitant fuzzy soft sets $(\widetilde{H}, Y)$ satisfying the following properties:

(1) $Y=\bigcup_{i \in I} Y_{i}$, and

(2) for any $p \in Y$ with $p \in \bigcap_{j \in J} Y_{j} \backslash \bigcup_{k \in I \backslash J} Y_{k}$, where $\emptyset \neq J \subseteq I, \widetilde{H}[p]=\bigcup_{j \in J} \widetilde{H}_{j}[p]$.

We write $\widetilde{\bigcup_{i \in I}}\left(\widetilde{H}_{i}, Y_{i}\right)=(\widetilde{H}, Y)$.

Theorem 5.12. The union of anti-hesitant fuzzy soft UP-subalgebras of $\mathrm{A}$ is an anti-hesitant fuzzy soft UPsubalgebra of $A$.

Proof. Let $\left(\widetilde{H}_{i}, Y_{i}\right)$ be an anti-hesitant fuzzy soft UP-subalgebra of $A$ for all $i \in I$. Then $\widetilde{H}_{i}\left[p_{i}\right]$ is an antihesitant fuzzy UP-subalgebras of $A$ for all $p_{i} \in Y_{i}$ and $i \in I$. Assume that $(\widetilde{H}, Y)$ is the union of $\left(\widetilde{H}_{i}, Y_{i}\right)$ for all $i \in I$. Then $Y=\bigcup_{i \in I} Y_{i}$. Let $p \in Y$.

If $p \in \bigcap_{i \in \mathrm{I}} Y_{i} \neq \emptyset$, then it follows from Theorem 5.6 that $(\widetilde{\mathrm{H}}, Y)$ is a p-anti-hesitant fuzzy soft UPsubalgebra of $A$.

If $p \in \bigcap_{j \in J} Y_{j} \backslash \bigcup_{k \in I \backslash J} Y_{k}$, where $\emptyset \neq J \subseteq I$, then $\widetilde{H}[p]=\bigcup_{j \in J} \widetilde{H}_{j}[p]$ and $p \in Y_{j}$ for all $j \in J$ but $p \notin Y_{k}$ for some $k \in I \backslash J$. Thus $\widetilde{\mathrm{H}}_{j}[p]$ is an anti-hesitant fuzzy UP-subalgebra of $A$ for all $j \in J$. By Theorem 4.32 , we have $\tilde{\mathrm{H}}[\mathrm{p}]=\bigcup_{j \in J} \widetilde{\mathrm{H}}_{j}[\mathrm{p}]$ is an anti-hesitant fuzzy UP-subalgebra of $A$. Therefore, $(\widetilde{\mathrm{H}}, Y)$ is a p-anti-hesitant fuzzy soft UP-subalgebra of $A$. Since $p$ is arbitrary, we know that $(\widetilde{H}, Y)$ is an anti-hesitant fuzzy soft UP-subalgebra of $A$.

In the same way as Theorem 5.12, we can use Theorem 5.7 (resp., Theorems 5.8, 5.9, 5.10) and Theorem 4.33 (resp., Theorems $4.34,4.35,4.31$ ) to prove that the union of anti-hesitant fuzzy soft UP-filters (resp., anti-hesitant fuzzy soft UP-ideals, anti-hesitant fuzzy soft strongly UP-ideals, hesitant fuzzy soft strongly 
UP-ideals) of $A$ is an anti-hesitant fuzzy soft UP-filter (resp., anti-hesitant fuzzy soft UP-ideal, anti-hesitant fuzzy soft strongly UP-ideal, hesitant fuzzy soft strongly UP-ideal) of $A$. By the way, we also confirm that the union of hesitant fuzzy soft UP-subalgebras (resp., hesitant fuzzy soft UP-filters, hesitant fuzzy soft UP-ideals) of $A$ is not a hesitant fuzzy soft UP-subalgebra (resp., hesitant fuzzy soft UP-filter, hesitant fuzzy soft UP-ideal) of $A$ in general.

Definition 5.13. Let $\left\{\left(\widetilde{H}_{i}, Y_{i}\right) \mid i \in I\right\}$ be a family of hesitant fuzzy soft sets over a reference set $X$. The intersection of $\left(\widetilde{H}_{i}, Y_{i}\right)$ is defined to be the hesitant fuzzy soft sets $(\widetilde{H}, Y)$ satisfying the following properties:

(1) $Y=\bigcap_{i \in I} Y_{i} \neq \emptyset$, and

(2) $\tilde{\mathrm{H}}[\mathrm{p}]=\bigcap_{i \in \mathrm{I}} \tilde{\mathrm{H}}_{i}[p]$ for all $p \in Y$.

We write $\widetilde{\bigcap_{i \in I}}\left(\widetilde{H}_{i}, Y_{i}\right)=(\widetilde{H}, Y)$.

Theorem 5.14. The intersection of hesitant fuzzy soft UP-subalgebras of $A$ is a hesitant fuzzy soft UP-subalgebra of A.

Proof. Let $\left(\widetilde{H}_{i}, Y_{i}\right)$ be a hesitant fuzzy soft UP-subalgebra of $A$ for all $i \in I$. Then $\widetilde{H}_{i}\left[p_{i}\right]$ is a hesitant fuzzy UP-subalgebras of $A$ for all $p_{i} \in Y_{i}$ and $i \in I$. Assume that $(\widetilde{H}, Y)$ is the intersection of $\left(\widetilde{H}_{i}, Y_{i}\right)$ for all $i \in I$ and let $p \in Y$. Then $p \in Y=\bigcap_{i \in I} Y_{i} \neq \emptyset$ and $\tilde{H}[p]=\bigcap_{i \in I} \widetilde{H}_{i}[p]$. Thus $p \in Y_{i}$ for all $i \in I$ and so $\widetilde{H}_{i}[p]$ is a hesitant fuzzy UP-subalgebras of $A$ for all $i \in I$. By Theorem 4.19 , we have $\tilde{H}[p]=\bigcap_{i \in I} \widetilde{H}_{i}[p]$ is a hesitant fuzzy UP-subalgebra of $A$. Therefore, $(\widetilde{H}, Y)$ is a p-hesitant fuzzy soft UP-subalgebra of $A$. Since $p$ is arbitrary, we know that $(\widetilde{H}, Y)$ is a hesitant fuzzy soft UP-subalgebra of $A$.

In the same way as Theorem 5.14, we can use Theorems 4.20,4.21, 4.22, and 4.26, respectively, to prove the following theorems.

Theorem 5.15. The intersection of hesitant fuzzy soft UP-filters of $A$ is a hesitant fuzzy soft UP-filter of A.

Theorem 5.16. The intersection of hesitant fuzzy soft UP-ideals of $A$ is a hesitant fuzzy soft UP-ideal of A.

Theorem 5.17. The intersection of hesitant fuzzy soft strongly UP-ideals of A is a hesitant fuzzy soft strongly UP-ideal of $A$.

Theorem 5.18. The intersection of anti-hesitant fuzzy soft strongly UP-ideals of $A$ is an anti-hesitant fuzzy soft strongly UP-ideal of A.

By Example 4.23 (resp., Example 4.24, 4.25), we can imply that the intersection of anti-hesitant fuzzy soft UP-subalgebras (resp., anti-hesitant fuzzy soft UP-filters, anti-hesitant fuzzy soft UP-ideals) of $A$ is not an anti-hesitant fuzzy soft UP-subalgebra (resp., anti-hesitant fuzzy soft UP-filter, anti-hesitant fuzzy soft UP-ideal) of $A$ in general.

Definition 5.19. Let $\left\{\left(\widetilde{H}_{i}, Y_{i}\right) \mid i \in I\right\}$ be a family of hesitant fuzzy soft sets over a reference set $X$. The extended intersection of $\left(\widetilde{H}_{i}, Y_{i}\right)$ is defined to be the hesitant fuzzy soft sets $(\widetilde{H}, Y)$ satisfying the following properties:

(1) $Y=\bigcup_{i \in I} Y_{i}$, and 
(2) for any $p \in Y$ with $p \in \bigcap_{j \in J} Y_{j} \backslash \bigcup_{k \in I \backslash J} Y_{k}$, where $\emptyset \neq J \subseteq I, \widetilde{H}[p]=\bigcap_{j \in J} \widetilde{H}_{j}[p]$.

We write $\widetilde{\bigcap_{i \in I}} e\left(\widetilde{H}_{i}, Y_{i}\right)=(\widetilde{H}, Y)$.

Theorem 5.20. The extended intersection of hesitant fuzzy soft UP-subalgebras of $A$ is a hesitant fuzzy soft UPsubalgebra of $\mathrm{A}$.

Proof. Let $\left(\widetilde{\mathrm{H}}_{i}, Y_{i}\right)$ be a hesitant fuzzy soft UP-subalgebra of $A$ for all $i \in \mathrm{I}$. Then $\widetilde{\mathrm{H}}_{i}\left[\mathrm{p}_{i}\right]$ is a hesitant fuzzy UP-subalgebras of $A$ for all $p_{i} \in Y_{i}$ and $i \in I$. Assume that $(\widetilde{H}, Y)$ is the extended intersection of $\left(\widetilde{H}_{i}, Y_{i}\right)$ for all $i \in I$. Then $Y=\bigcup_{i \in I} Y_{i}$. Let $p \in Y$.

If $p \in \bigcap_{i \in \mathrm{I}} Y_{i} \neq \emptyset$, then it follows from Theorem 5.16 that $(\widetilde{\mathrm{H}}, \mathrm{Y})$ is a $p$-hesitant fuzzy soft UP-subalgebra of $A$.

If $p \in \bigcap_{j \in J} Y_{j} \backslash \bigcup_{k \in I \backslash J} Y_{k}$, where $\emptyset \neq J \subseteq I$, then $\widetilde{H}[p]=\bigcap_{j \in J} \widetilde{H}_{j}[p]$ and $p \in Y_{j}$ for all $j \in J$ but $p \notin Y_{k}$ for some $k \in I \backslash J$. Thus $\widetilde{H}_{j}[p]$ is a hesitant fuzzy UP-subalgebra of $A$ for all $j \in J$. By Theorem 4.19, we have $\widetilde{\mathrm{H}}[\mathrm{p}]=\bigcap_{j \in J} \widetilde{\mathrm{H}}_{j}[\mathrm{p}]$ is a hesitant fuzzy UP-subalgebra of $A$. Therefore, $(\widetilde{\mathrm{H}}, \mathrm{Y})$ is a p-hesitant fuzzy soft UP-subalgebra of $A$. Since $p$ is arbitrary, we know that $(\widetilde{H}, Y)$ is a hesitant fuzzy soft UP-subalgebra of A.

In the same way as Theorem 5.20, we can use Theorem 5.15 (resp., Theorems 5.16, 5.17, 5.18) and Theorem 4.20 (resp., Theorems 4.21, 4.22, 4.23) to prove that the extended intersection of hesitant fuzzy soft UP-filters (resp., hesitant fuzzy soft UP-ideals, hesitant fuzzy soft strongly UP-ideals, anti-hesitant fuzzy soft strongly UP-ideals) of $A$ is a hesitant fuzzy soft UP-filter (resp., a hesitant fuzzy soft UP-ideal, a hesitant fuzzy soft strongly UP-ideal, an anti-hesitant fuzzy soft strongly UP-ideal) of $A$. By the way, we also confirm that the extended intersection of hesitant fuzzy soft UP-subalgebras (resp., hesitant fuzzy soft UP-filters, hesitant fuzzy soft UP-ideals) of $A$ is not a hesitant fuzzy soft UP-subalgebra (resp., hesitant fuzzy soft UP-filter, hesitant fuzzy soft UP-ideal) of $A$ in general.

Definition 5.21. Let $\left\{\left(\widetilde{H}_{i}, Y_{i}\right) \mid i \in I\right\}$ be a family of hesitant fuzzy soft sets over a reference set $X$. The AND of $\left(\widetilde{H}_{i}, Y_{i}\right)$ is defined to be the hesitant fuzzy soft sets $(\widetilde{H}, Y)$ satisfying the following properties:

(1) $Y=\prod_{i \in I} Y_{i}$, and

(2) $\tilde{\mathrm{H}}\left[\left(p_{i}\right)_{i \in \mathrm{I}}\right]=\bigcap_{i \in \mathrm{I}} \tilde{\mathrm{H}}_{i}\left[p_{i}\right]$ for all $\left(p_{i}\right)_{i \in I} \in Y$.

We write $\widetilde{\bigwedge}_{i \in \mathrm{I}}\left(\widetilde{\mathrm{H}}_{i}, Y_{i}\right)=(\widetilde{\mathrm{H}}, \mathrm{Y})$.

Theorem 5.22. The AND of hesitant fuzzy soft UP-subalgebras of A is a hesitant fuzzy soft UP-subalgebra of A.

Proof. Let $\left(\widetilde{H}_{i}, Y_{i}\right)$ be a hesitant fuzzy soft UP-subalgebra of $A$ for all $i \in I$ and let $p_{i} \in Y_{i}$ for all $i \in I$. Then $\widetilde{\mathrm{H}}_{i}\left[p_{i}\right]$ is a hesitant fuzzy UP-subalgebras of $A$. Assume that $(\widetilde{\mathrm{H}}, Y)$ is the AND of $\left(\widetilde{\mathrm{H}}_{i}, Y_{i}\right)$ for all $i \in I$. Then $Y=\prod_{i \in I} Y_{i}$ and $\widetilde{H}\left[\left(p_{i}\right)_{i \in I}\right]=\bigcap_{i \in I} \widetilde{H}_{i}\left[p_{i}\right]$. By Theorem 4.19, we have $\widetilde{H}\left[\left(p_{i}\right)_{i \in I}\right]=\bigcap_{i \in I} \widetilde{H}_{i}\left[p_{i}\right]$ is a hesitant fuzzy UP-subalgebra of $A$. Therefore, $(\widetilde{H}, Y)$ is a $\left(p_{i}\right)_{i \in I}$-hesitant fuzzy soft UP-subalgebra of $A$. Since $\left(p_{i}\right)_{i \in I}$ is arbitrary, we know that $(\widetilde{H}, Y)$ is a hesitant fuzzy soft UP-subalgebra of $A$. 
In the same way as Theorem 5.22, we can use Theorem 4.20 (resp., Theorems 4.21 and 4.22) to prove that the AND of hesitant fuzzy soft UP-filters (resp., hesitant fuzzy soft UP-ideals and hesitant fuzzy soft strongly UP-ideals) of $A$ is a hesitant fuzzy soft UP-filter (resp., hesitant fuzzy soft UP-ideal, hesitant fuzzy soft strongly UP-ideal) of $A$.

Definition 5.23. Let $\left\{\left(\widetilde{H}_{i}, Y_{i}\right) \mid i \in I\right\}$ be a family of hesitant fuzzy soft sets over a reference set $X$. The OR of $\left(\widetilde{H}_{i}, Y_{i}\right)$ is defined to be the hesitant fuzzy soft sets $(\widetilde{H}, Y)$ satisfying the following properties:

(1) $Y=\prod_{i \in I} Y_{i}$, and

(2) $\tilde{\mathrm{H}}\left[\left(p_{i}\right)_{i \in \mathrm{I}}\right]=\bigcup_{i \in \mathrm{I}} \tilde{\mathrm{H}}_{i}\left[p_{i}\right]$ for all $\left(p_{i}\right)_{i \in I} \in Y$.

We write $\widetilde{\bigvee}\left(\widetilde{\mathrm{H}}_{i}, Y_{i}\right)=(\widetilde{\mathrm{H}}, \mathrm{Y})$.

Theorem 5.24. The OR of anti-hesitant fuzzy soft UP-subalgebras of $\mathrm{A}$ is an anti-hesitant fuzzy soft UP-subalgebra of $A$.

Proof. Let $\left(\widetilde{H}_{i}, Y_{i}\right)$ be an anti-hesitant fuzzy soft UP-subalgebra of $A$ for all $i \in I$ and let $p_{i} \in Y_{i}$ for all $i \in \mathrm{I}$. Then $\widetilde{\mathrm{H}}_{i}\left[p_{i}\right]$ is a hesitant fuzzy UP-subalgebras of $A$. Assume that $(\widetilde{\mathrm{H}}, Y)$ is the OR of $\left(\widetilde{\mathrm{H}}_{i}, Y_{i}\right)$ for all $i \in I$. Then $Y=\prod_{i \in I} Y_{i}$ and $\widetilde{H}\left[\left(p_{i}\right)_{i \in I}\right]=\bigcup_{i \in I} \widetilde{H}_{i}\left[p_{i}\right]$. By Theorem 4.32, we have $\widetilde{H}\left[\left(p_{i}\right)_{i \in I}\right]$ is an antihesitant fuzzy UP-subalgebra of $A$. Therefore, $(\widetilde{H}, Y)$ is a $\left(p_{i}\right)_{i \in I}$-anti-hesitant fuzzy soft UP-subalgebra of $A$. Since $p$ is arbitrary, we know that $(\widetilde{H}, Y)$ is an anti-hesitant fuzzy soft UP-subalgebra of $A$.

In the same way as Theorem 5.24, we can use Theorem 4.33 (resp., Theorems 4.34 and 4.35) to prove that the OR of anti-hesitant fuzzy soft UP-filters (resp., anti-hesitant fuzzy soft UP-ideals and anti-hesitant fuzzy soft strongly UP-ideals) of $A$ is an anti-hesitant fuzzy soft UP-filter (resp., anti-hesitant fuzzy soft UP-ideal and anti-hesitant fuzzy soft strongly UP-ideal) of $A$.

\section{Acknowledgment}

This work was supported by the Unit of Excellence, University of Phayao. The authors would also like to thank the anonymous referee for giving many helpful suggestion on the revision of present paper.

\section{References}

[1] M. I. Ali, F. Feng, X. Y. Liu, W. K. Min, M. Shabir, On some new operations in soft set theory, Comput. Math. Appl., 57 (2009), 1547-1553. 1

[2] M. A. Ansari, A. Haidar, A. N. A. Koam, On a graph associated to UP-algebras, Math. Comput. Appl., 23 (2018), 12 pages. 2

[3] K. V. Babitha, S. J. John, Hesitant fuzzy soft sets, J. New Res. Sci., 3 (2013), 98-107. 1, 3.6

[4] T. Guntasow, S. Sajak, A. Jomkham, A. Iampan, Fuzzy translations of a fuzzy set in UP-algebras, J. Indones. Math. Soc., 23 (2017), 1-19. 2.5, 2

[5] A. Iampan, A new branch of the logical algebra: UP-algebras, J. Algebra Relat. Topics, 5 (2017), 35-54. 1, 2.1, 2, 2.4, 2, 2.5

[6] A. Iampan, Introducing fully UP-semigroups, Discuss. Math. Gen. Algebra Appl., 38 (2018), 297-306. 2, 2.4

[7] Y. B. Jun, K. J. Lee, S.-Z. Song, Hesitant fuzzy bi-ideals in semigroups, Commun. Korean Math. Soc., 30 (2015), 143-154. 1, 4

[8] W. Kaijae, P. Poungsumpao, S. Arayarangsi, A. Iampan, UP-algebras characterized by their anti-fuzzy UP-ideals and anti-fuzzy UP-subalgebras, Ital. J. Pure Appl. Math., 36 (2016), 667-692. 1

[9] B. Kesorn, K. Maimun, W. Ratbandan, A. Iampan, Intuitionistic fuzzy sets in UP-algebras, Ital. J. Pure Appl. Math., 34 (2015), 339-364. 1 
[10] P. K. Maji, R. Biswas, A. R. Roy, Fuzzy soft sets, J. Fuzzy Math., 9 (2001), 589-602. 1

[11] P. K. Maji, R. Biswas, A. R. Roy, Intuitionistic fuzzy soft sets, J. Fuzzy Math., 9 (2001), 677-692. 1

[12] P. K. Maji, R. Biswas, A. R. Roy, Soft set theory, Comput. Math. Appl., 45 (2003), 555-562. 1

[13] P. K. Maji, R. Biswas, A. R. Roy, On intuitionistic fuzzy soft sets, J. Fuzzy Math., 12 (2004), 669-683. 1

[14] D. Molodtsov, Soft set theory-first results, Comput. Math. Appl., 37 (1999), 19-31. 1

[15] P. Mosrijai, A. Iampan, Anti-type of hesitant fuzzy sets on UP-algebras, Eur. J. Pure Appl. Math., 11 (2018), $976-1002$. $1,3.4,3,3.5$

[16] P. Mosrijai, A. Iampan, Hesitant fuzzy soft sets over UP-algebras, Ann. Fuzzy Math. Inform., 16 (2018), 317-331. 1, 3.7

[17] P. Mosrijai, A. Iampan, Hesitant fuzzy soft sets over UP-algebras by means of anti-type, Anal. Fuzzy Math. Inform., 16 (2018), 317-331. 1

[18] P. Mosrijai, A. Iampan, A new branch of bialgebraic structures: UP-bialgebras, J. Taibah Univ. Sci., 13 (2019), $450-459$. 3.8

[19] P. Mosrijai, W. Kamti, A. Satirad, A. Iampan, Hesitant fuzzy sets on UP-algebras, Konuralp J. Math., 5 (2017), 268-280. 1, 3.2, 3, 3.3

[20] P. Mosrijai, A. Satirad, A. Iampan, Partial constant hesitant fuzzy sets on UP-algebras, J. New Theory, 22 (2018), 39-50. 1

[21] C. Prabpayak, U. Leerawat, On ideals and congruences in KU-algebras, Sci. Magna, 5 (2009), 54-57. 1

[22] D. A. Romano, Proper UP-filters of UP-algebra, Univ. J. Math. Appl., 1 (2018), 98-100. 1

[23] A. Satirad, P. Mosrijai, A. Iampan, Formulas for finding UP-algebras, Int. J. Math. Comput. Sci., 14 (2019), $403-409$. 2

[24] A. Satirad, P. Mosrijai, A. Iampan, Generalized power UP-algebras, Int. J. Math. Comput. Sci., 14 (2019), 17-25. 2.2, $2.3,2$

[25] A. Satirad, P. Mosrijai, W. Kamti, A. Iampan, Level subsets of a hesitant fuzzy set on UP-algebras, Ann. Fuzzy Math. Inform., 14 (2017), 279-302. 1

[26] T. Senapati, Y. B. Jun, K. P. Shum, Cubic set structure applied in UP-algebras, Discrete Math. Algorithms Appl., 10 (2018), 23 pages. 1

[27] T. Senapati, G. Muhiuddin, K. P. Shum, Representation of UP-algebras in interval-valued intuitionistic fuzzy environment, Ital. J. Pure Appl. Math., 38 (2017), 497-517. 1

[28] J. Somjanta, N. Thuekaew, P. Kumpeangkeaw, A. Iampan, Fuzzy sets in UP-algebras, Ann. Fuzzy Math. Inform., 12 (2016), 739-756. 1, 2.5

[29] M. Songsaeng, A. Iampan, N-fuzzy UP-algebras and its level subsets, J. Algebra Relat. Topics, 6 (2018), 1-24. 1

[30] S. Sripaeng, K. Tanamoon, A. Iampan, On anti Q-fuzzy UP-ideals and anti Q-fuzzy UP-subalgebras of UP-algebras, J. Inf. Optim. Sci., 39 (2018), 1095-1127. 1

[31] K. Tanamoon, S. Sripaeng, A. Iampan, Q-fuzzy sets in UP-algebras, Songklanakarin J. Sci. Technol., 40 (2018), 9-29. 1

[32] V. Torra, Hesitant fuzzy sets, Int. J. Intell. Syst., 25 (2010), 529-539. 1, 3.1, 4, 4.1

[33] F. Q. Wang, X. Li, X. H. Chen, Hesitant fuzzy soft set and its applications in multicriteria decision making, J. Appl. Math., 2014 (2014), 10 pages. 1, 5, 5.1, 5.2, 5.3, 5.4

[34] X. B. Yang, T. Y. Lin, Y. Li, D. J. Yu, Combination of interval-valued fuzzy set and soft set, Comput. Math. Appl., 58 (2009), 521-527. 1 